\title{
Shear localization in dynamic deformation of materials: microstructural evolution and self-organization
}

\author{
Marc A. Meyers ${ }^{a, *}$, Vitali F. Nesterenko a, Jerry C. LaSalvia ${ }^{\text {b }}$, Qing Xue ${ }^{\text {a }}$ \\ a Department of Mechanical and Aerospace Engineering, University of California, San Diego, Mail Code 0411, 9500 Gilman Drive, La Jolla, \\ CA 92093-0411, USA \\ b Army Research Laboratory, Aberdeen Proving Ground, Aberdeen, MD 21005-5069, USA
}

\begin{abstract}
The plastic deformation of crystalline and non-crystalline solids incorporates microscopically localized deformation modes that can be precursors to shear localization. Shear localization has been found to be an important and sometimes dominant deformation and fracture mode in metals, fractured and granular ceramics, polymers, and metallic glasses at high strains and strain rates. Experiments involving the collapse of a thick walled cylinder enable controlled and reproducible application of plastic deformation at very high strain rates to specimens. These experiments were supplemented by hat-shaped specimens tested in a compression Hopkinson bar. The initiation and propagation of shear bands has been studied in metals ( $\mathrm{Ti}$, Ta, Ti-6Al-4V, and stainless steel), granular and prefractured ceramics $\left(\mathrm{Al}_{2} \mathrm{O}_{3}\right.$ and $\left.\mathrm{SiC}\right)$, a polymer (teflon) and a metallic glass $\left(\mathrm{Co}_{58} \mathrm{Ni}_{10} \mathrm{Fe}_{5} \mathrm{Si}_{11} \mathrm{~B}_{16}\right)$. The first aspect that was investigated is the microstructural evolution inside the shear bands. A fine recrystallized structure is observed in $\mathrm{Ti}, \mathrm{Cu}, \mathrm{Al}-\mathrm{Li}$, and $\mathrm{Ta}$, and it is becoming clear that a recrystallization mechanism is operating. The fast deformation and short cooling times inhibit grain-boundary migration; it is shown, for the first time, that a rotational mechanism, presented in terms of dislocation energetics and grain-boundary reorientation, can operate within the time of the deformation process. In pre-fractured and granular ceramics, a process of comminution takes place when the particles are greater than a critical size $a_{\mathrm{c}}$. When they are smaller than $a_{\mathrm{c}}$, particle deformation takes place. For the granular SiC, a novel mechanism of shear-induced bonding was experimentally identified inside the shear bands. For all materials, shear bands exhibit a clear self-organization, with a characteristic spacing that is a function of a number of parameters. This self-organization is analyzed in terms of fundamental material parameters in the frame of Grady-Kipp (momentum diffusion), Wright-Ockendon, and Molinari (perturbation) models. (C) 2001 Elsevier Science B.V. All rights reserved.
\end{abstract}

Keywords: Dynamic deformation; Plastic deformation; Crystalline solids

\section{Introduction}

Professor A.S. Argon's contributions in the field of mechanical behavior of materials cover an extraordinarily broad range and are characterized by a great depth of thought, as well as rigor in conception and computation [1-5]. A recurring theme in Professor Argon's work has been shear localization, especially in polymers and glassy metals. He demonstrated that the nucleation and growth of shear is the prevalent deformation mechanism in glassy media. Deng et al. [6] performed molecular dynamics calculations that show

\footnotetext{
* Corresponding author. Tel.: +1-858-5344719; fax: + 1-8585345698.

E-mail address: mameyers@mae.ucsd.edu (M.A. Meyers).
}

clearly localized regions of intense shear as the main entities responsible for plastic deformation of glasses. Bulatov and Argon [7-9] present analytical calculations which show that strain localization is an integral part of inelastic deformation of non-crystalline materials. The Argon concept of 'local inelastic transformation' is shown in Fig. 1(a) [6]. Small regions, marked by ellipses in the figure, undergo considerably higher strain than the globally imposed deformation. These strain ellipsoids are equivalent to transformation centers. As deformation proceeds, the local inelastic transformations organize themselves in bands. Fig. 1(b) and (c), from Bulatov and Argon [7], show these bands, which have a characteristic spacing and organize themselves into a pattern. Shear localization in glassy metals was clearly demonstrated experimentally by Pampillo and Chen [10], among others. 
The objectives of this contribution are: (a) to illustrate the importance of shear localization at high strain rates; (b) to present a mechanism for the microstructural evolution within shear bands; and (c) to propose that their self-organization plays a key role in the global deformation. The generality of localization will be demonstrated by showing illustrated examples in metals, ceramics, polymers, and metallic glasses.

\section{Experimental approach}

The shear bands described in this paper were generated by dynamic loading of the specimens and can be considered adiabatic. The following experimental techniques were used:

1. The hat-shaped specimen, in which one single band is forced by prescribed initial conditions. This technique was introduced by Meyer and Manwaring [11] and was used successfully for steel [12], $\mathrm{Cu}$ [13-15], Ti [16], Ta [17-19], and Al-Li alloys [20,21]. Fig. 2(a) shows the hat-shaped specimen placed in a Hopkinson pressure bar. The strain, upon activation of the pressure bar, is localized in a thin region, and strain rates of $10^{4} \mathrm{~s}^{-1}$ and shear strains of up to 5-6 are easily and reproducibly achieved. It should be pointed out that this localization is forced, and not spontaneously generated.

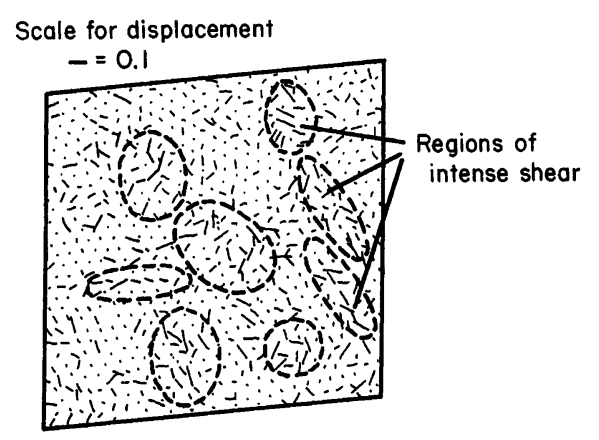

(a)

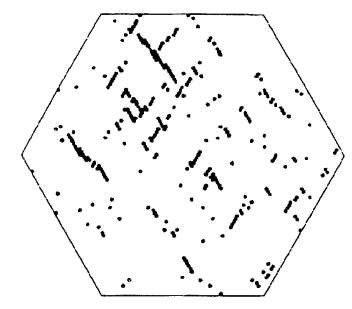

(b)

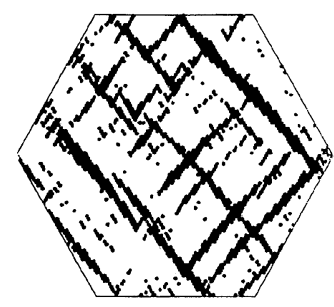

(c)
Fig. 1. (a) Displacement fields of atoms(indicated by magnitude and direction of lines) when assemblage of non-crystalline atomic array is subjected to a shear strain of $5 \times 10^{-2}$; molecular dynamics simulation(from Deng et al. [6]), (b) and (c) Regions of plastic deformation organizing themselves into bands as plastic stain is increased (from Bulatov and Argon [9])
2. The thick-walled cylinder technique, in which a thick-walled cylinder is collapsed (totally, or to prescribed strains) by explosives placed co-axially with the cylinder. The material under investigation (metal, metallic glass, ceramic or granular medium) is placed within copper tubes (both outside and inside) and the energy of the explosive produces the implosion of the cylinder at initial collapse velocities on the order of $200 \mathrm{~m} \mathrm{~s}^{-1}$. Fig. 2(b) shows the basic configuration. The material (M) is encased in copper tubes, and the external energy is provided by an explosive which is initiated at the top. The shear band pattern is shown after the collapse of the cylinder in Fig. 2(c). This technique was developed by Nesterenko et al. [22,23]. The inner copper tube serves as a stopper, and strains in the inner surface can be prescribed by the size of the orifice and other dimensions.

\section{Microstructural evolution in shear localization}

\subsection{Metals}

It is impossible to resolve the details of microstructure evolution within shear bands by optical or scanning electron microscopy. Some of the earliest detailed observations by TEM were made by Grebe et al. [24] and by Meyers and Pak [25] on shear bands produced in $\mathrm{Ti}-6 \mathrm{Al}-4 \mathrm{~V}$ and commercial purity titanium, respectively. Fig. 3(a) shows a TEM micrograph of a shear band in Ti observed under an accelerating voltage of 1 $\mathrm{MeV}$. The shear band has a thickness of approximately $10 \mu \mathrm{m}$ and cuts through the micrograph in a diagonal manner. The electron diffraction patterns inside and outside of the shear band are radically different; outside the band, the characteristic pattern for a single crystalline orientation is clear. Inside the band, a ring-like pattern, produced by many crystallographic orientations, is apparent. At a higher magnification, the detailed structure inside of the shear band is revealed (Fig. 3(b)). It consists of equiaxed grains, with diameters of $0.05-0.2 \mu \mathrm{m}$. The dislocation density is relatively low. This remarkable feature led to the suggestion by Meyers and Pak [23] that the structure was due to dynamic recrystallization. The microscopic observations made within areas of intense plastic deformation in copper, tantalum, and an $\mathrm{Al}-\mathrm{Li}$ alloy, produced by the hat shaped technique, are shown in Fig. 4(a)-(c), respectively. Dynamic recrystallization in shock-conditioned copper was first observed by Andrade et al. [14]; in tantalum, it was first observed by Meyers et al. [17], and Nesterenko et al. [18] and confirmed by NematNasser et al. [26]. In Al-Li alloys, Chen and Vecchio [20] and $\mathrm{Xu}$ et al. [21] observed dynamic recrystallization. The microstructures for these different crystal 


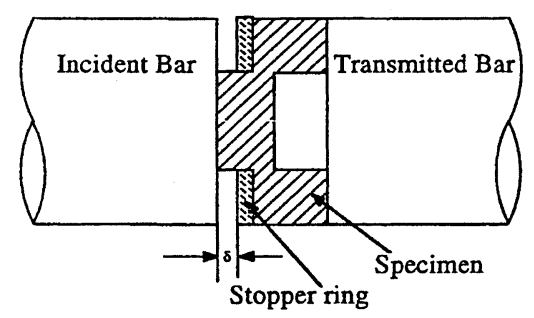

(a)

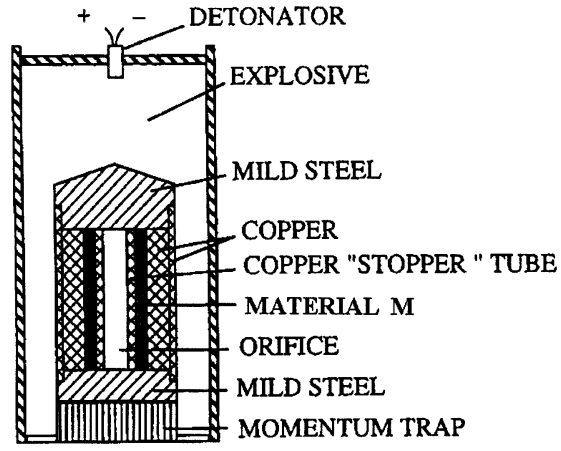

(b)
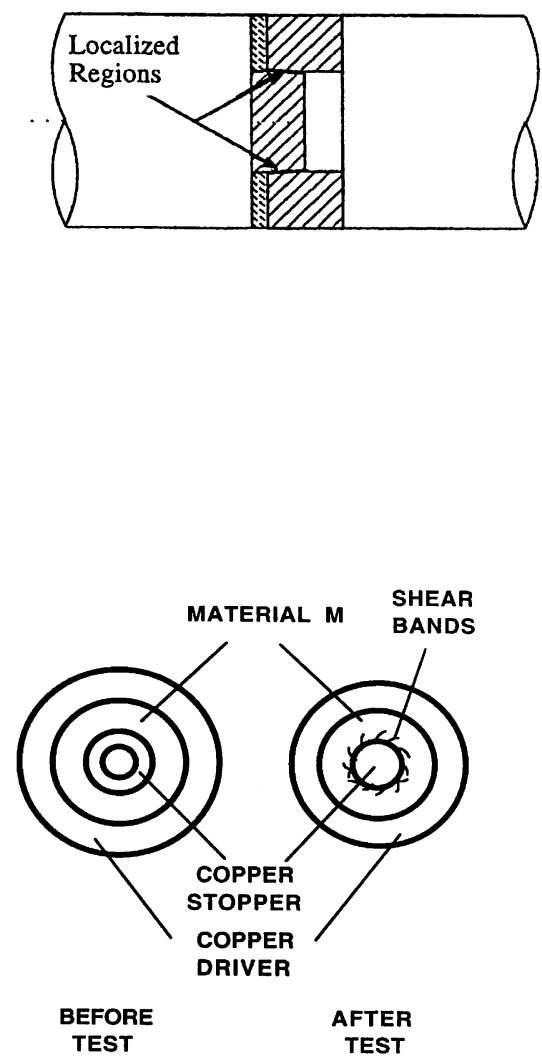

(c)

Fig. 2. Two experimental techniques applied for large strain, high strain-rate deformation of materials: (a) hat-shaped specimen(initial and final configurations); (b) thick-walled cylinder method; (c) initial and final configurations (cross section) in thick-walled cylinder technique.

structures (HCP, FCC, BCC) are remarkably similar: approximately equiaxed micrograins with diameters between 0.1 and $0.3 \mu \mathrm{m}$. The grains have a relatively low dislocation density, and the dihedral angles at grain boundary triple points $\left(\sim 120^{\circ}\right)$ indicate that the boundaries have energies consistent with high angles. Three such triple points are marked by arrows in Fig. 4. For tantalum and $8090 \mathrm{Al}-\mathrm{Li}$ alloy, a dark-field micrograph is shown in Fig. 4(b) and (c), respectively, to better illustrate the morphology of the grains and the significant misorientations. The microstructures shown in Fig. 4 are clearly indicative of recrystallization. Since the thermo-mechanical history of the shear localization regions is rather complex, one asks the obvious questions:

1. Do the observed recrystallization features occur during or after plastic deformation?

2. What is the mechanism of recrystallization?

First, calculations will establish for titanium, copper, and tantalum, the plastic strains required to produce temperatures at which diffusional processes become important; this temperature is set as $0.4 T_{\mathrm{m}}$, where $T_{\mathrm{m}}$ is the melting temperature. This can be readily done by assuming adiabatic conditions for the deformation process and using a constitutive equation that incorporates strain hardening, strain-rate hardening, and thermal softening. One possible constitutive equation, useful due to its simplicity, is the Johnson-Cook [27] equation, which expresses the flow stress, $\sigma$, as a function of a number of parameters with a modified thermal softening component (for ease of integration):

$\sigma=\left(\sigma_{0}+k \varepsilon^{n}\right)\left[1+C_{1} \ln \left(\frac{\dot{\varepsilon}}{\dot{\varepsilon}_{0}}\right)\right] \mathrm{e}^{-\lambda\left(T-T_{\mathrm{r}}\right)}$

where $\sigma_{0}$ and $\dot{\varepsilon}_{0}$ are the yield stress and the strain rate at the reference temperature $T_{\mathrm{r}}$, respectively; $k$ and $n$ are strain-hardening parameters; $C_{1}$ is a strain-rate hardening parameter; $T_{\mathrm{r}}$ is a reference temperature; $\varepsilon$ is the plastic strain; $\dot{\varepsilon}$ is the strain rate; and $\lambda$ is a thermal softening parameter. The temperature is obtained from the conversion of the deformation energy to internal energy using the parameter $\beta$ (rate of conversion of deformation energy into heat, usually taken as 0.9 ) and integrating: 


$$
\begin{aligned}
\mathrm{d} T= & \left(\frac{\beta}{\rho C}\right) \sigma \mathrm{d} \varepsilon \mathrm{T} \\
= & T_{0}+\frac{1}{\lambda} \ln \left[\mathrm{e}^{-\lambda\left(T_{0}-T_{\mathrm{r}}\right)}\right. \\
& \left.+\left(\frac{\beta \lambda}{\rho C}\right)\left[1+C_{1} \ln \left(\frac{\dot{\varepsilon}}{\dot{\varepsilon}_{0}}\right)\right]\left[\sigma_{0}+\left(\frac{k}{n+1}\right) \varepsilon^{n}\right] \varepsilon\right]
\end{aligned}
$$

The heat capacity is $C$ and the density is $\rho$. The temperatures, normalized with respect to the corresponding melting temperature, are plotted in Fig. 5(a) as a function of plastic strain for titanium, copper (shock hardened), and tantalum. The true strain $\left(\varepsilon_{\mathrm{t}}\right)$ required to reach the recrystallization range is material dependent. For titanium, the value is reached for $\varepsilon_{\mathrm{t}}=1.2$; for copper (shock-hardened to increase its flow stress) the strain is approximately 1.9; and for tantalum, the required strain is much higher, approximately 4.4. The strains generated in the hat-shaped specimen are sufficient to create these favorable thermal regimes for $\mathrm{Cu}$, Ti and the Al- Li alloy. Meyers et al. [17] obtained the temperature of dynamic recrystallization in isolated areas using the hat-shaped specimen at an engineering shear strain $\gamma=4\left(\varepsilon_{\mathrm{t}}=1.2\right)$ and, recently, Nemat-Nasser et al. [26] obtained recrystallization using the hat-shaped specimen, but at $\gamma=9$ $\left(\varepsilon_{\mathrm{t}}=1.9\right)$. However, this is the upper limit of deformation accessible using the hat specimen, and the thick-walled cylinder technique is more appropriate at these high strains. The strain rate imparted by both methods is fairly similar, approximately $4 \times 10^{4} \mathrm{~s}^{-1}$.

The cooling times can be estimated from a computational heat-transfer analysis. The results are shown in Fig. 5(b). Shear-band thicknesses were taken as 10, 200, and $100 \mu \mathrm{m}$ for titanium, copper, and tantalum, respectively. These values are obtained from experimental measurements. If the recrystallized structure forms after deformation, a migrational mechanism is required, because of the absence of mechanical assistance. It is possible to estimate the size of the recrystallized grains assuming migrational recrystallization. To a first approximation, it is assumed that the following simple grain growth equation can be used [28]:

$\Delta d=k_{0} \Delta t^{1 / n} \exp \left(-\frac{Q}{2 R T(t)}\right)$

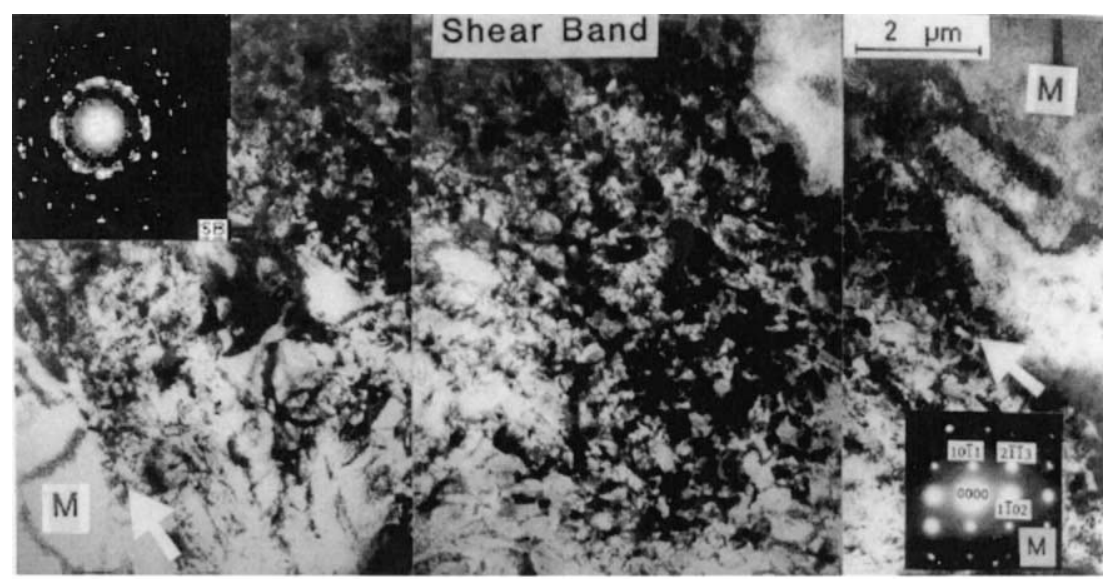

(a)

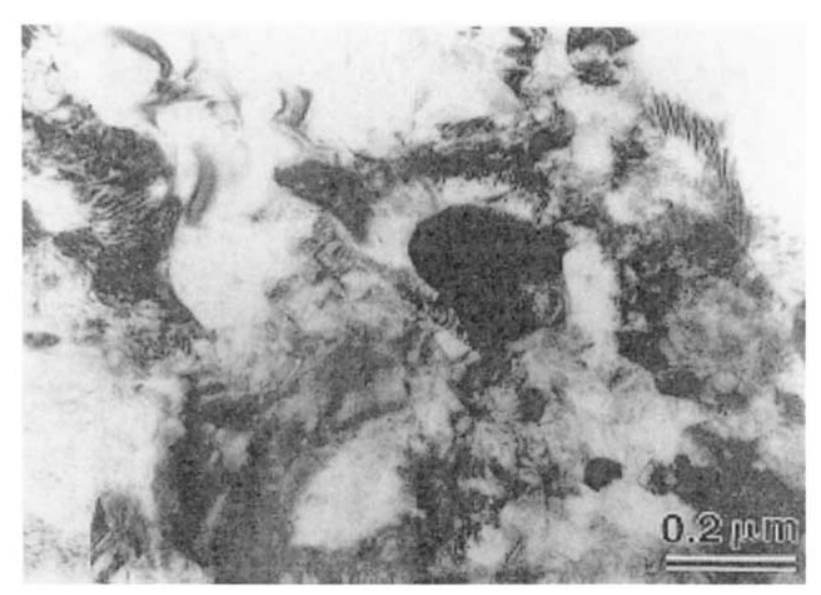

(b)

Fig. 3. (a) TEM micrograph of shear band in titanium arrows represent the boundary between matrix (M) and shear band ; (b) Microstructural details within band. 

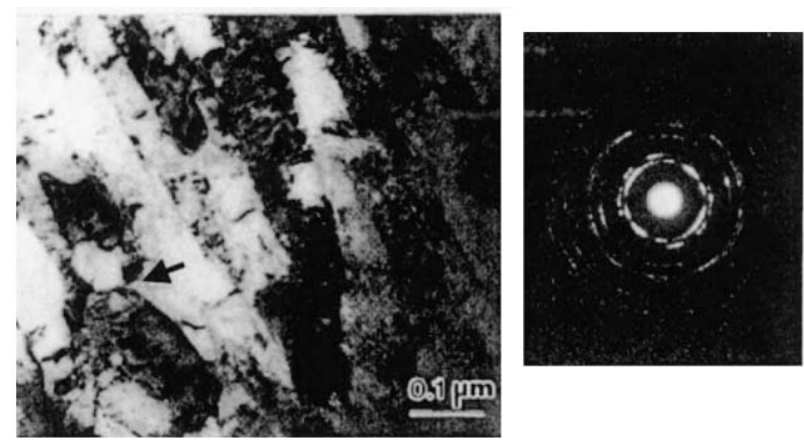

(a)
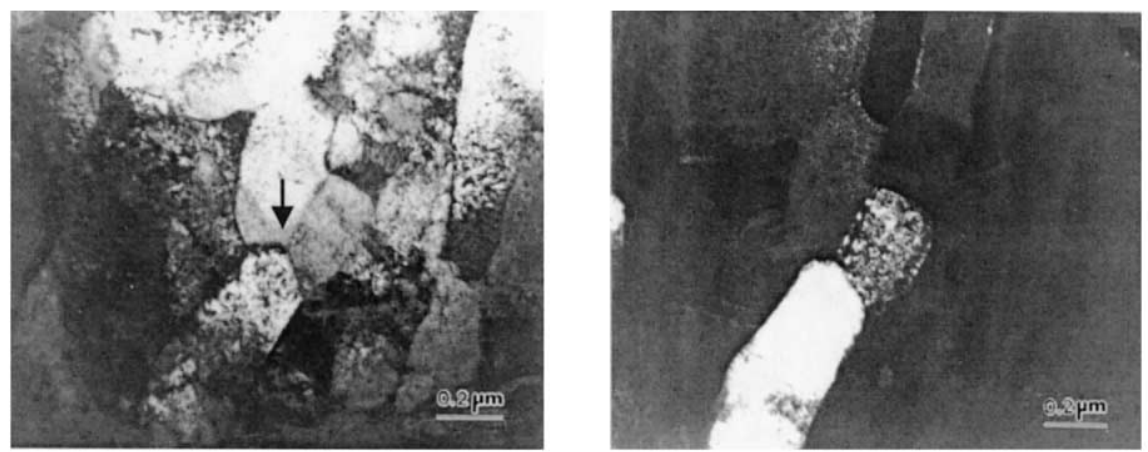

(b)
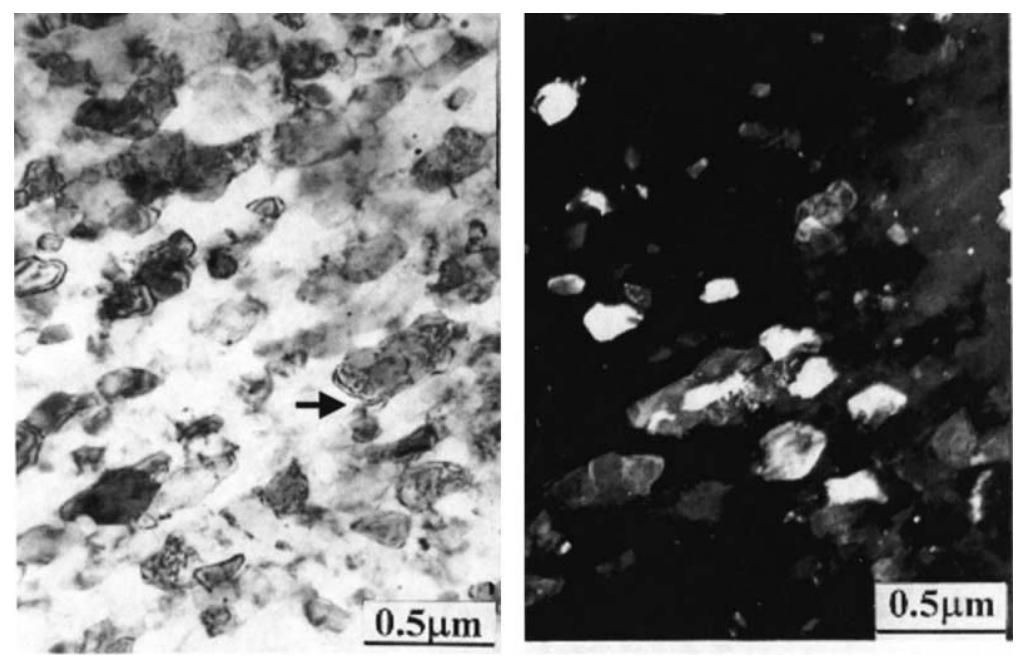

Fig. 4. TEM micrographs of microcrystalline structure within shear band in (a) copper (Andrade et al.[14]), (b) tantalum(Nesterenko et al.[18] and (c) $8090 \mathrm{Al}-\mathrm{Li}$ alloy $(\mathrm{Xu}$ et al.[21])

By summation, over the entire time, one has:

$d \cong k_{0} \sum_{i=0}^{N}\left[\exp \left(-\frac{Q}{2 R T\left(t_{i}\right)}\right)\right] \Delta t^{1 / n}$

where $\Delta d$ is the change in the instantaneous grain size $d, k_{0}$ is a rate constant, $Q$ is the activation energy for grain growth, $\Delta t$ is the change in time $t$, and $T$ is the absolute temperature. The activation energy for grain boundary migration can be taken as the activation energy for self-diffusion or for recrystallization. The pre-exponential factor $k$ and exponent $1 / n$ can be estimated from experimental results reported in the literature [29-32]. The exponent $n$ varies between 2 and 10 depending upon impurity content (e.g. $n=2$ for ultra pure metals). The final grain size is obtained by numerically integrating Eq. (3a) during cooling. This formalism was applied to the three metals and grain sizes were obtained. The activation energies, pre-exponential factors, exponents $1 / n$, and predicted grain sizes are given in Table 1. The values for $\mathrm{Cu}, \mathrm{Ta}$ and $\mathrm{Ti}$ fluctuate 


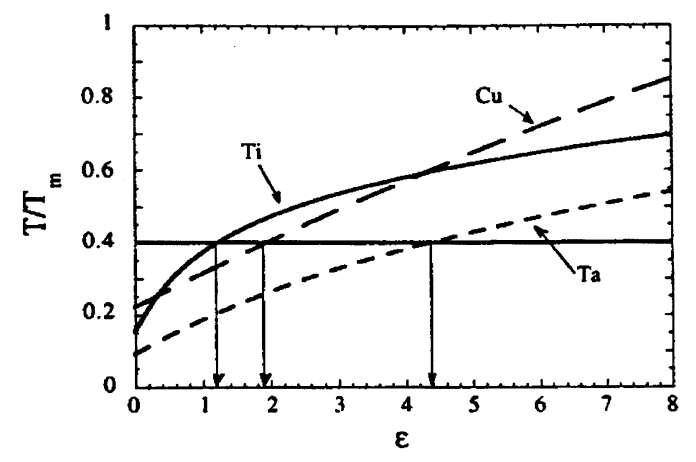

(a)

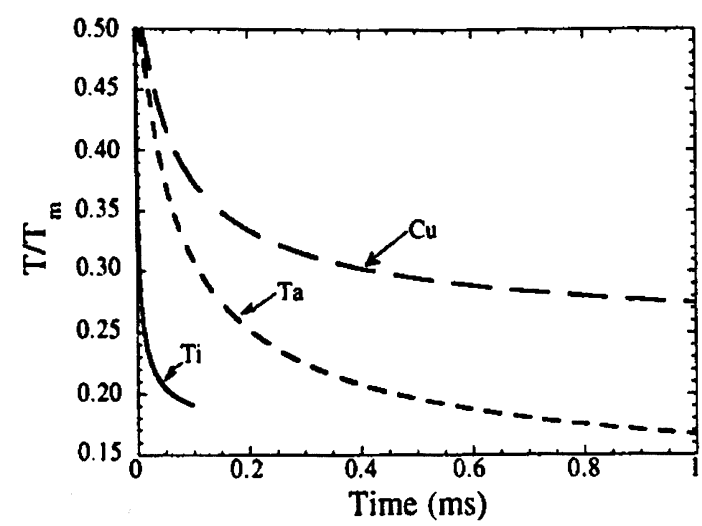

(b)

Fig. 5. (a) Normalized temperature as a function of plastic strain for titanium, copper, and tantalum (b) Shear-band cooling curves for titanium, copper, and tantalum

around $2 \sim 9 \times 10^{-4} \mu \mathrm{m}$; this is clearly unrealistic and it is concluded that migrational recrystallization does not occur during cooling. It can be seen that the calculated grain sizes assuming migrational recrystallization during cooling are inconsistent with the observed results. It is also instructive to establish the deformation time. The deformation time $t$ is simply given by the total strain divided by the strain-rate. These times are, respectively, for titanium, copper, and tantalum: $t=4 \times 10^{-5} ; 2.5 \times$ $10^{-5}$; and $10 \times 10^{-5} \mathrm{~s}$. Only a fraction of this deformation time occurs at a temperature sufficient to induce recrystallization. Hence, the time for recrystallization, $t_{\mathrm{r}}$, is given by:

$t_{\mathrm{r}}=t-t_{\mathrm{c}}=t-\frac{\varepsilon_{\mathrm{c}}}{\dot{\varepsilon}}$ $t_{\mathrm{c}}$ is the time required to heat the specimen to the critical recrystallization temperature and $\varepsilon_{\mathrm{c}}$ is the critical strain at which $T / T_{\mathrm{m}}=0.4$. These times range, for the specimens analyzed, from 2 to $5 \times 10^{-5} \mathrm{~s}$. This time is lower by one order of magnitude than the cooling time (see Fig. 5(b)). This would also exclude migrational recrystallization. Thus a migrational mechanism alone cannot occur either in deformation or cooling and cannot account for the recrystallized structure.

Derby [33] classifies dynamic recrystallization mechanisms into rotational and migrational types. Rotational recrystallization needs concurrent plastic deformation. It is well documented for geological materials such as quartz [34], halite [35], marble [36], and sodium nitrate [37]. The observations in shear bands of copper [14], tantalum [18] and titanium [25] are suggestive of this mechanism. Indeed Meyers and Pak [25] suggested a rotational recrystallization mechanism in titanium, and this was corroborated by Meyers et al. [15], Andrade et al. [14] for copper, and Nesterenko et al. [18], for tantalum. Fig. 6 shows the primary features of the process by which rotational dynamic recrystallization is thought to occur. As can be seen from this figure, a homogeneous distribution of dislocations rearranges itself into elongated dislocation cells (i.e. dynamic recovery). As the deformation continues and as the misorientation increases, these cells become elongated subgrains. Eventually, the elongated subgrains break up into approximately equiaxed micrograins. Fig. 7 shows a structure produced in tantalum subjected to a strain of 5.5. These elongated subgrains are a characteristic feature of copper and titanium subjected to subrecrystallization strains. These elongated structures correspond to stage(c) in Fig. 6 and are seen in many metals subjected to high strains, as reported by Gil Sevillano et al. [38], among others. Hughes and Hansen [39] reported rotations of $30-45^{\circ}$ at medium and large strains (cold rolling reductions from 70 to $90 \%$ ). Hughes and Kumar [40] made detailed TEM observation on heavily deformed Ta and found evolution from Configuration $\mathrm{b}-\mathrm{c}$ in Fig. 6. Hynes et al. [41] modeled this process of grain boundary rotation using a bicrystal and calculating numerically the strains using crystal plasticity theory.

The formation of these elongated sub-grains is analyzed below. This process of rotational recrystallization was modeled by Meyers et al. [42] using dislocation

Table 1

Grain growth parameters for $\mathrm{Cu}(\mathrm{FCC}), \mathrm{Ta}(\mathrm{BCC})$, and $\mathrm{Ti}(\mathrm{HCP})$

\begin{tabular}{llllll}
\hline Material & $k_{0}\left(\mathrm{~ms}^{1 / n}\right)$ & $Q(\mathrm{~kJ} / \mathrm{mol})$ & $n$ & $d(\mu \mathrm{m})$ & Source \\
\hline $\mathrm{Cu}$ & 72.5 & $255-326$ & 2 & $1.8 \times 10^{-4}$ & Weinig and Machlin [32] \\
$\mathrm{Ta}$ & 19.7 & 300 & 10 & $8.6 \times 10^{-4}$ & Vandermeer and Snyder [30],Kraschenko and Statsenko [31] \\
$\mathrm{Ti}$ & 4.3 & 322 & 1 & $3.9 \times 10^{-4}$ & Okazaki and Conrad [29] \\
\hline
\end{tabular}




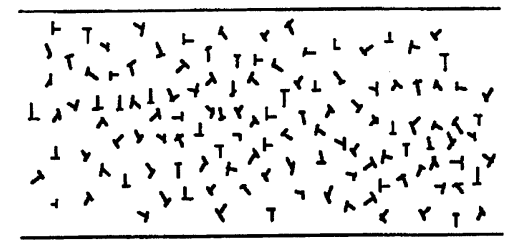

(a)

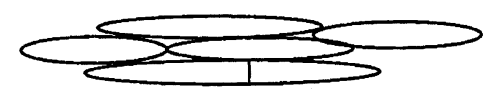

(c)

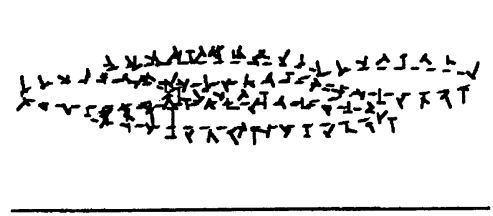

(b)

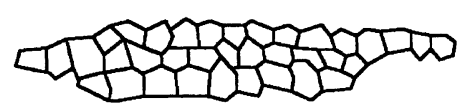

(e)

Fig. 6. Schematic illustration of microstructural evolution during high-strain-rate deformation. (a) Randomly distributed dislocations; (b) Elongated dislocation cell formation (i.e. dynamic recovery); (c) Elongated subgrain formation; (d) Initial break-up of elongated subgrains; and (e) Recrystallized microstructure( from Nesterenko et al. [18]).

energetics. The strain energy for a random dislocation configuration (a in Fig. 6) is given, per unit volume of material, by

$\mathrm{E}_{1}=\rho_{\mathrm{d}}\left(\frac{\mathrm{A} \mu \mathrm{b}^{2}}{4 \pi}\right) \ln \left(\frac{\alpha}{2 b \rho_{\mathrm{d}}^{1 / 2}}\right)$

where $A$ is a constant depending upon the character of the dislocation, $\mu$ is the shear modulus, $b$ is the magnitude of the Burgers vector, $\alpha$ is a constant which takes in account the core energy of the dislocation, $\rho_{\mathrm{d}}$ is the dislocation density. If the dislocations are arranged into cells, the energy is altered. A simple manner to represent the elongated cells is to assume that they are ellipsoidal. This corresponds to Configuration $b$ in Fig. 6. The cell walls can be modeled as tilt boundaries. For an ellipsoid with aspect ratio $k$, the surface area $S$ and volume $V$ are given by:

$$
\begin{aligned}
& S=2 \pi f(k) W^{2} \quad V=\frac{4}{3} \pi k W^{3} \\
& f(k)=1+\frac{k^{2}}{\sqrt{k^{2}-1}} \sin ^{-1}\left(\frac{\sqrt{k^{2}-1}}{k}\right)
\end{aligned}
$$

where $2 W$ is the cell width. A dislocation density, $\rho_{\mathrm{d}}$, can be assumed to be completely arranged in the cell walls. Eq. (7) represents the energy if dislocations are arranged into cells:

$$
E_{2}=\rho_{\mathrm{d}}\left(\frac{\mathrm{A} \mu \mathrm{b}^{2}}{4 \pi}\right) \ln \left[\frac{e \alpha}{4 \pi b}\left(\frac{S}{V}\right) \frac{1}{\rho_{\mathrm{d}}}\right]
$$

The dislocation spacing, $D_{\mathrm{d}}$, at the tilt (cell) boundaries is related to the misorientation, $\theta$, by:

$$
D_{\mathrm{d}}=\frac{b}{2 \sin (\theta / 2)} \cong \frac{b}{\theta}
$$

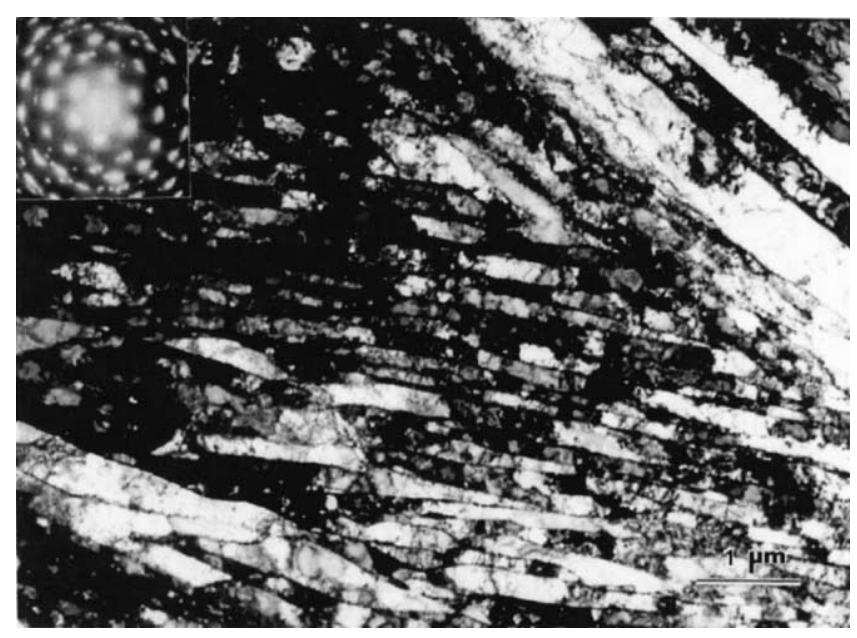

Fig. 7. TEM micrograph showing elongated subgrains in Ta subjected to a shear strain of 5.5 at a strain rate of $5 \times 10^{4} \mathrm{~s}^{-1}$. 


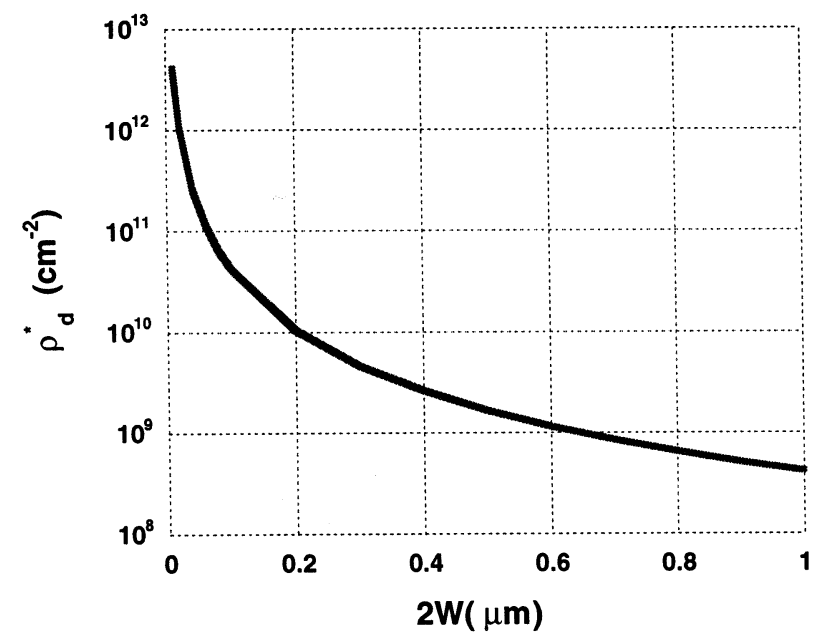

(b)

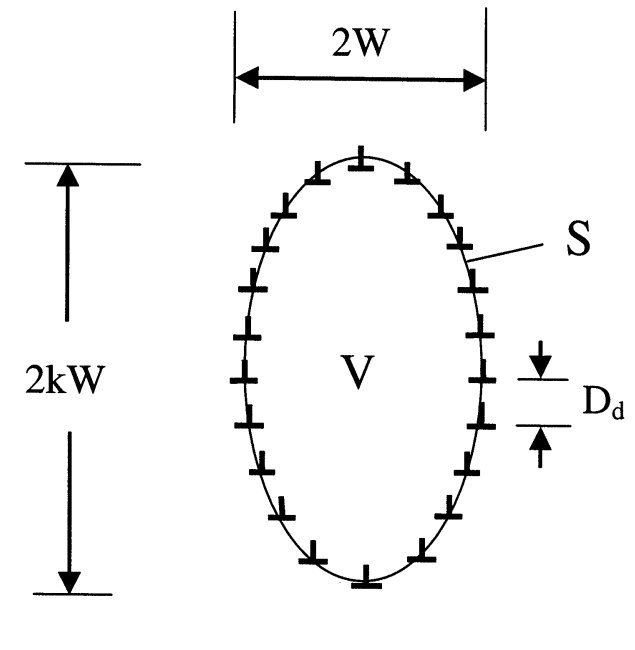

(a)

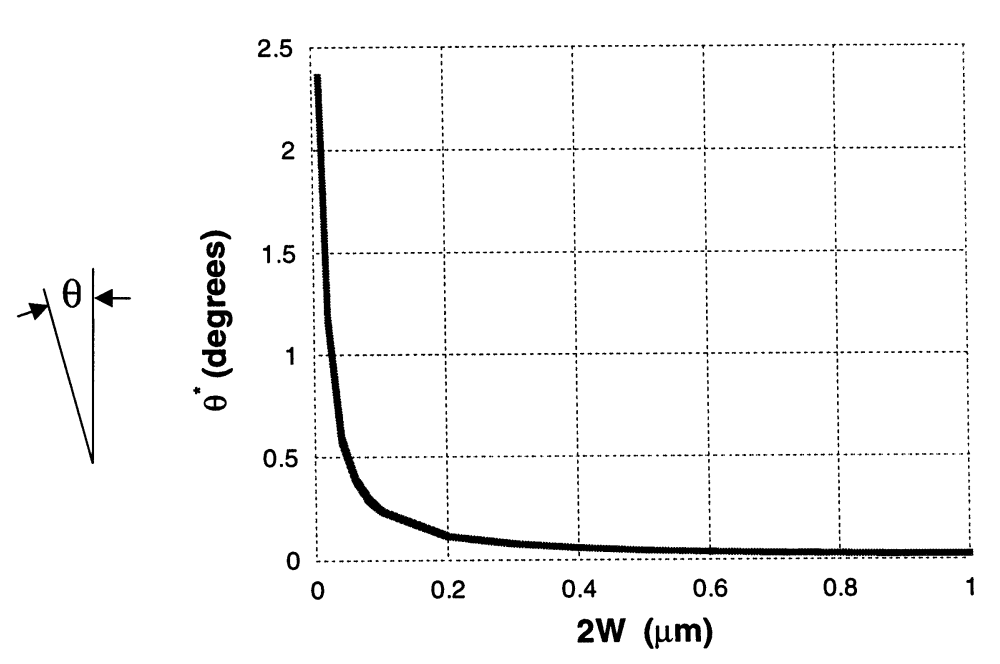

(c)

Fig. 8. Critical dislocation density $\rho^{*}$ and resulting misorientation angle $\theta^{*}$ as a function of cell width $2 \mathrm{~W}$.

Eqs. (5) and (7) give the total energy per unit volume due to the dislocations in Configurations a and $\mathrm{b}$, respectively. For low dislocation densities, $E_{1}>E_{2}$. The condition $E_{1}=E_{2}$ gives the following expressions for the critical dislocation density $\rho_{\mathrm{d}}^{*}$ and misorientation angle $\theta^{*}$ at which Configuration $\mathrm{b}$ (Fig. 16) is of equal energy to Configuration a:

$\rho_{\mathrm{d}}^{*}=\left[\frac{3 e}{4 \pi}\left(\frac{f(k)}{k}\right)\left(\frac{1}{W}\right)\right]^{2}$

$\theta^{*}=\frac{3}{4}\left(\frac{e}{\pi}\right)^{2}\left(\frac{f(k)}{k}\right)\left(\frac{b}{W}\right)$

It can also be shown that the energy difference between Configurations $\mathrm{a}$ and $\mathrm{b}$ is a maximum for $k \rightarrow+\infty$, from which $f(k) / k=\pi / 2$. Substitution of this condition into Eqs. (8) and (9) gives the following expressions for the critical dislocation density $\rho_{\mathrm{d}}^{*}$ and resulting misorientation angle $\theta^{*}$ :

$\rho_{\mathrm{d}}^{*}=\left[\frac{3 e}{8}\left(\frac{1}{W}\right)\right]^{2}$

$\theta^{*}=\frac{3 e^{2}}{8 \pi}\left(\frac{b}{W}\right)$

Eqs. (10) and (11) are plotted in Fig. 8 as a function of cell width for tantalum $(b=2.333$ A for $\{112\}\langle 111\rangle$ dislocations, the favored slip system for this metal in dynamic deformation). The predicted misorientation angles for the observed cell widths are in excellent agreement with measured values. It is interesting to note that maximum energy difference between configu- 
rations $\mathrm{a}$ and $\mathrm{b}$ is given for an aspect ratio $k \rightarrow+\infty$. This is also consistent with observation that once the dislocation cells initially form, they are very long.

These results are in agreement with the TEM observations. A typical cell width varies between 0.1 and 0.3 $\mu \mathrm{m}$, and cell formation occurs at a strain of approximately 1.8 , with a temperature rise of $400 \mathrm{~K}$. This corresponds, approximately, to a dislocation density of $10^{10} \mathrm{~cm}^{-2}$. The predictions of Fig. 8 provide similar values for $\rho^{*}$ and $\theta^{*}$ for dislocation densities in the range $10^{10}-10^{11} \mathrm{~cm}^{-2}$.

After the dislocation sub-grains have formed, there must be a net increase in dislocation density as plastic deformation continues. If this increase in dislocation density is primarily accommodated at cell walls, the misorientation between cells is expected to increase if cells do not dissociate (consistent with observations). It will be shown bellow that the misorientation angle $\theta$ can be expressed in terms of the dislocation density and the plastic deformation.

After dislocation cells have formed, dislocation density $\rho$ is given by:

$\rho_{\mathrm{d}}=\frac{\theta}{W b}$

Since $\rho=1 / W D$ and $D_{\mathrm{d}}=b / \theta$ for small dislocation spacing in low angle grain boundaries, one has, from Eq. (10)

$\frac{1}{W}=\frac{8}{3 e} \rho_{\mathrm{d}}^{* 1 / 2}$

Therefore,

$\rho_{\mathrm{d}}=\frac{8}{3 e} \frac{\theta}{b} \rho_{\mathrm{d}}^{* 1 / 2}$

$\frac{\rho_{\mathrm{d}}}{\rho_{\mathrm{d}}^{*}}=\frac{8}{3 e} \frac{\theta}{b \rho_{\mathrm{d}}^{* 1 / 2}}$

From Eqs. (10) and (11), eliminating $W$ :

$\rho_{\mathrm{d}}^{*}=\left[\frac{\pi \theta^{*}}{e b}\right]^{2}$

Substitution into Eq. (12) yields:

$\frac{\rho_{\mathrm{d}}}{\rho_{\mathrm{d}}^{*}}=\frac{8}{3 \pi}\left(\frac{\theta}{\theta^{*}}\right)$

But $8 / 3 \pi \approx 1$; thus

$\frac{\rho_{\mathrm{d}}}{\rho_{\mathrm{d}}^{*}} \approx \frac{\theta}{\theta^{*}}$

For strain, applying the Orowan equation and Eq. (10):

$\varepsilon^{*}=\rho_{\mathrm{d}}^{*} b \Delta_{1}=\frac{9 e^{2}}{64}\left(\frac{b \Delta_{1}}{W^{2}}\right)$ where $\Delta$ is the average distance traveled by each dislocation. After dislocation cells have formed,

$\varepsilon=\rho_{\mathrm{d}} b \Delta_{2}$

$\frac{\varepsilon}{\varepsilon^{*}}=\left(\frac{\Delta_{2}}{\Delta_{1}}\right)\left(\frac{\rho_{\mathrm{d}}}{\rho_{\mathrm{d}}^{*}}\right)=\left(\frac{\Delta_{2}}{\Delta_{1}}\right) \frac{8}{3 \pi}\left(\frac{\theta}{\theta^{*}}\right)$

$\frac{\theta}{\theta^{*}}=\left(\frac{\Delta_{1}}{\Delta_{2}}\right)\left(\frac{3 \pi}{8}\right)\left(\frac{\varepsilon}{\varepsilon^{*}}\right)$

For

$\frac{\Delta_{1}}{\Delta_{2}} \cong 1 \cdot \frac{\theta}{\theta^{*}} \approx\left(\frac{\varepsilon}{\varepsilon^{*}}\right)$

Thus:

$\left(\frac{\theta}{\theta^{*}}\right) \cong\left(\frac{\rho_{\mathrm{d}}}{\rho_{\mathrm{d}}^{*}}\right) \cong\left(\frac{\varepsilon}{\varepsilon^{*}}\right)$

The ratios between the misorientations, dislocation densities and plastic strains are approximately the same. Cells evolve into sub-grains as the misorientation increases. Beyond $\theta^{*}=2-2.5^{\circ}$, the adjacent grains define random boundaries. Random boundaries constitute barriers for dislocations. As observations show, the elongated cells eventually break-up longitudinally. This must be due to a slow-down in the ability of the cell walls to accommodate dislocations or due to capillary type of instability. This leads to a reorganization of dislocations into low-energy boundaries perpendicular to the cell walls. The breakup of the subgrains to form micrograins is the second stage of this process and requires thermal energy and diffusional processes (albeit at a very small scale). This corresponds to the transition $\mathrm{d}-\mathrm{e}$ in Fig. 6. Small grain-boundary rotations are required and the driving force is the minimization of the overall energy as well as torques applied to the triple points.

It is interesting to note that as early as 1961, Sabato and Cahn [43] observed recrystallized shear bands in uranium subjected to a $70 \%$ reduction by forging. Part of the recrystallized grains formed as a result of static, post-deformation recrystallization; however, it is very possible that grains too small to resolve by optical microscopy were the result of dynamic recrystallization. The shiny, 'transformed' appearance reported by many investigators in shear bands in steels is the result of a fine recrystallized structure with an associated dissolution of carbides, resulting in an increased resistance to etching $[12,44,45]$. These features were in the past mistakingly identified as phase transformation products.

The relaxation of the broken-down elongated subgrains into an equiaxed micro-crystalline structure can occur by minor rotations of the grain boundaries lying along the original elongated boundaries, as shown in Fig. 9. If each longitudinal grain boundary segment $A B$ rotates to A'B' by an angle $\theta=30^{\circ}$, an equiaxed structure will be produced. This is illustrated in Fig. 9(b). It 
will be shown how this can be accomplished. The flux of atoms along the grain boundary can occur at rates that are orders of magnitude higher than in the bulk. The activation energy for grain boundary diffusion is approximately one half of that for lattice diffusion, and at $T / T_{\mathrm{m}}=0.5$, the ratio between grain boundary coefficient of diffusion, $D_{\mathrm{GB}}$ and lattice coefficient of diffusion, $D_{\mathrm{L}}$ is:

$10^{7}<\frac{D_{\mathrm{GB}}}{D_{\mathrm{L}}}<10^{8}$

These results are reported for FCC metals by Sutton and Balluffi [46] and Shewmon [47]. The activation energy for grain-boundary diffusion is approximately one half of that for lattice diffusion; hence, the difference increases as the temperature decreases.

A general form of Fick's law, expressed in terms of a potential energy gradient, can be obtained [46]. The force $\vec{F}$, acting on a particle, is:

$\vec{F}=\nabla V$

where $\nabla V$ is the gradient of the potential energy field. The mean diffusion velocity $\vec{v}$ is the product of the mobility $M$ by this force:

$\vec{v}=M \vec{F}$

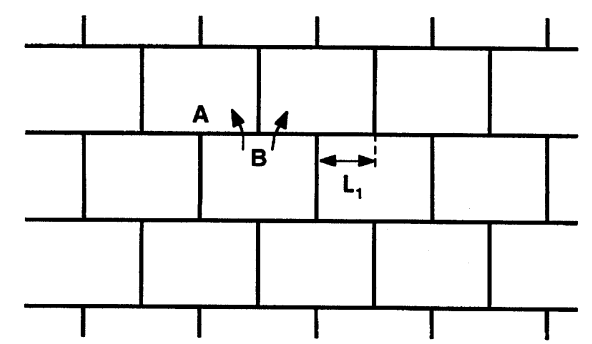

(a)

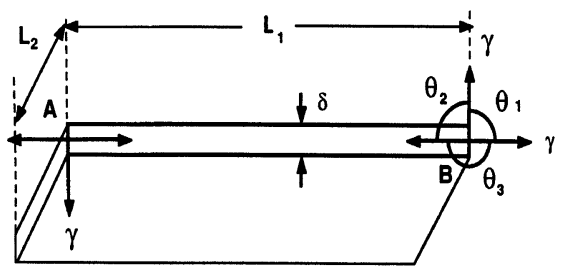

(c)
The mobility of the diffusing species is defined as (it has units of velocity/force):

$M=\frac{D}{k T}$

where $D$ is the diffusion coefficient. The flux along a grain boundary with thickness $\delta$ and depth $L_{2}$ is equal to (the cross-sectional area is $L_{2} \delta$ ):

$J=L_{2} \delta C M F=\left(\frac{L_{2} \delta D C_{\mathrm{m}}}{k T}\right) F$

$C_{\mathrm{m}}$, the concentration of the mobile species, is expressed in terms of mass per unit volume.

The rotation of the boundaries is driven by the minimization of the interfacial energy. The force exerted by the grain boundaries is represented in Fig. 9(d) and is equal to:

$F=\gamma\left(1-2 \cos \frac{\theta_{3}}{2}\right) L_{2}$

The relationship between the grain boundary rotation and the volume flow, $\mathrm{d} V$, required through the mid-point of the grain boundary AB is (this is computed per thickness, $L_{2}$ ):

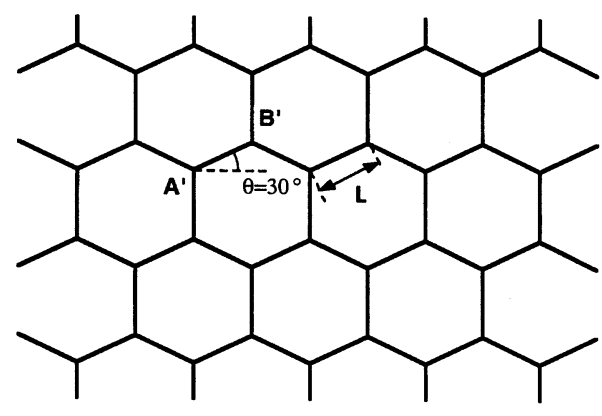

(b)

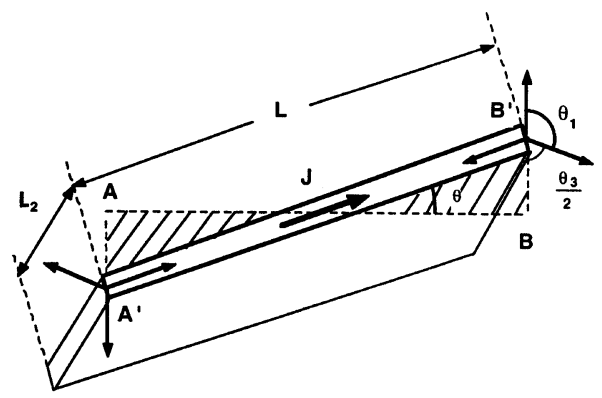

(d)

Fig. 9. Rotation of grain boundaries leading to equiaxed configuration: (a) broken down subgrains; (b) rotation of boundaries; (c) a grain boundary $A B$ under effect of interfacial energies; (d) material flux through grain boundary diffusion and rotation of AB to A'B'. 

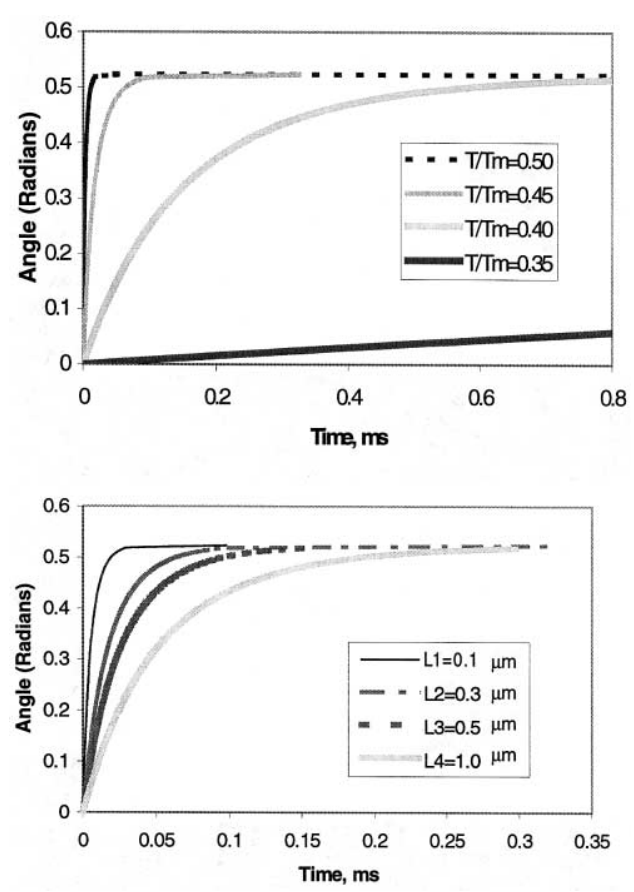

Fig. 10. Angle of rotation of micro-grain boundary AB as a function of time for (a) different temperatures and (b) different lengths $(0.1$, $0.3,0.5$, and $1 \mu \mathrm{m})$ at $T / T_{\mathrm{m}}=0.45$.

Table 2

Parameters for Grain Boundary Rotation

\begin{tabular}{lll}
\hline Parameter & Value & Source \\
\hline Grain boundary energy, $\gamma$ & $0.625 \mathrm{~J} / \mathrm{m}^{2}$ & Murr[48] \\
Grain boundary thickness, & $0.5 \mathrm{~nm}$ & Sutton and \\
$\delta$ & & Balluffi[46] \\
Grain boundary diffusion & $10^{-12.3} \mathrm{~m}^{2} / \mathrm{s}$ & Shewmon[47] \\
coefficient, $D_{\mathrm{GB}}$ & $T / T_{\mathrm{m}}=0.5$ & \\
& $10^{-13} \mathrm{~m}^{2} / \mathrm{s}$ & \\
& $T / T_{\mathrm{m}}=0.45$ & \\
& $10^{-14} \mathrm{~m}^{2} / \mathrm{s}$ & \\
& $T / T_{\mathrm{m}}=0.4$ & \\
& $10^{-15.5} \mathrm{~m}^{2} / \mathrm{s}$ & \\
& $T / T_{\mathrm{m}}=0.35$ & \\
\hline
\end{tabular}

$\frac{L^{2} d \theta}{4 L_{2}}=\mathrm{d} V$

$\mathrm{d} V$ is the volume transferred and $L$ is the instantaneous length of the segment A'B' (Fig. 9(d)). But:

$L=\frac{L_{1}}{\cos \theta}$

where $L_{1}$ is the initial length, or AB. Thus

$\frac{\mathrm{d} \theta}{\mathrm{d} t}=\frac{4 \cos ^{2} \theta}{L_{2} L_{1}^{2}} \frac{\mathrm{d} V}{\mathrm{~d} t}=\frac{4 \cos ^{2} \theta}{L_{2} L_{1}^{2} \rho} \frac{\mathrm{d} m}{\mathrm{~d} t}$ $\mathrm{d} m / \mathrm{d} t$ is the mass change, which is the rate of volume change multiplied by $\rho$, the density.

The rate of mass change is the flux, and we have, substituting Eq. (26):

$\frac{\mathrm{d} \theta}{\mathrm{d} t}=\frac{4 \cos ^{2} \theta}{L_{2} L_{1}^{2} \rho} J=\frac{4 \cos ^{2} \theta}{L_{2} L_{1}^{2} \rho}\left(\frac{L_{2} \delta D C_{\mathrm{m}}}{k T}\right) F$

Eq. (27) is substituted into Eq. (31) by noting that.

$\frac{\theta_{3}}{2}=\frac{\pi}{2}-\theta$

$\frac{\mathrm{d} \theta}{\mathrm{d} t}=\frac{4 \cos ^{2} \theta}{L_{1}^{2} \rho} \frac{\delta D C_{\mathrm{m}}}{k T} \gamma(1-2 \sin \theta) L_{2}$

One considered equiaxed grains and $L_{2} \approx L_{1}$; one also considered one sole diffusing species, and $C_{\mathrm{m}}=$ $\rho$ :

$\frac{4 \delta D \gamma}{L_{1} k T} t=\int_{0}^{\theta} \frac{\mathrm{d} \theta}{\cos ^{2} \theta(1-2 \sin \theta)}$

Integrating by parts one arrives at:

$\int_{0}^{\theta} \frac{\mathrm{d} \theta}{\cos ^{2} \theta(1-2 \sin \theta)}=\frac{\tan \theta}{(1-2 \sin \theta)}$
$\left.-\int_{0}^{\theta} \frac{2 \sin \theta}{(1-2 \sin \theta)^{2}} \mathrm{~d} \theta=\frac{4 \delta D \gamma}{L_{1} k T} t\right)$

And finally:

$\frac{\tan \theta-\frac{2}{3} \cos \theta}{(1-2 \sin \theta)}+\frac{4}{3 \sqrt{3}} \ln \frac{\tan \frac{\theta}{2}-2-\sqrt{3}}{\tan \frac{\theta}{2}-2+\sqrt{3}}+\frac{2}{3}$
$-\frac{4}{3 \sqrt{3}} \ln \frac{2+\sqrt{3}}{2-\sqrt{3}}=\frac{4 \delta D \gamma}{L_{1} k T} t$

The predictions of Eq. (36) for copper are shown in Fig. 10. In Fig. 10(a) the temperature is varied from 0.35 to $0.5 T_{\mathrm{m}}$ for a subgrain size of $0.3 \mu \mathrm{m}$; in Fig. 10(b) the sub-grain size, $L$, is varied from 0.1 to $1 \mu \mathrm{m}$. The parameters used in Eq. (36) are given in Table 2.

The rate of rotation decreases with increasing $\theta$ and asymptotically approaches $30^{\circ}$ as $t \rightarrow \infty$. The calculations predict significant rotations of the boundary within the deformation time $\left(\sim 5 \times 10^{-5} \mathrm{~s}\right.$ or 0.05 $\mathrm{ms}$ ) at temperatures of 0.45 and $0.5 T_{\mathrm{m}}$, for micrograin sizes of $0.1-0.3 \mu \mathrm{m}$. Thus, the second stage of rotational recrystallization can also take place during plastic deformation. This does not exclude the possibility of reorientation/accommodation of the grain boundaries during cooling.

\subsection{Ceramics}

Both fractured and granular ceramics undergo shear localization in deformation, and this is the di- 
rect consequence of avoiding dilatation that accompanies homogeneous deformation. This dilatation of an idealized fragmented solid or fully dense granular aggregate is shown in Fig. 11; hexagonal arrays are represented in Fig. 11(a). In the absence of confining pressure, the shear stress $\tau$ will produce movement of the hexagonal blocks (Fig. 11(b)) and this results in displacements $\chi$, which produce dilatation (hatched areas marked). With a superimposed hydrostatic component of the stress, this dilatation creates additional friction stress which results from the pressure rise under confined deformation. A simple yet elegant solution found by nature is to localize deformation in a single region and to minimize dilatation. This is shown in Fig. 11(c) and (d). The dilatation is now localized in the shear band.

Shear localization is an important deformation mode in the quasi-static mechanical response of granular materials; it has been widely investigated both from analytical and experimental point of view [49-60]. Rudnicki and Rice [49] developed a general formulation for shear localization in pressure-sensitive dilatant materials, of which granular materials are a special case. There are no reports, to the authors' knowledge, of shear localization under high-strain-rate loading of granular materials, before the work of Nesterenko, Meyers and Chen $[61,62]$ on alumina.

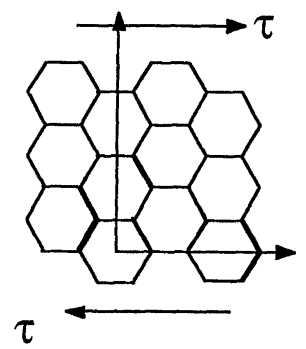

(a)

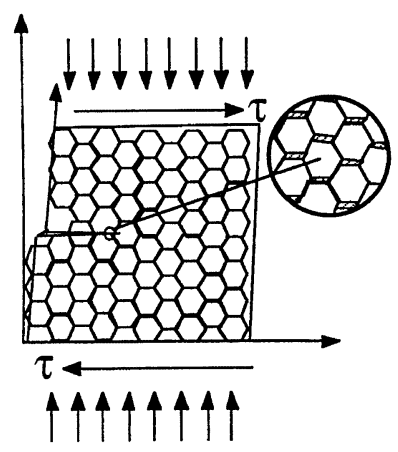

(c)

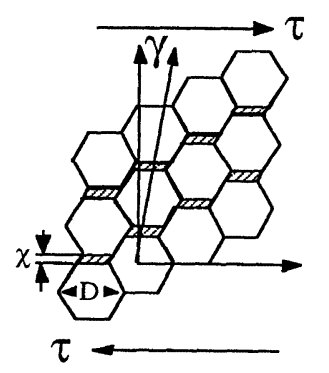

(b)

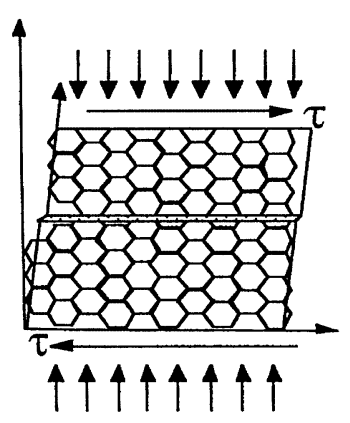

(d)
Fig. 11. $(a, b)$ Homogeneous deformation of hexagonal array of fragments, causing dilatation, (c, d) Shear localization under confined shear deformation of hexagonal arrays, avoiding dilatation (from Shih et al. [63]).
Shear localization under dynamic loading was observed in both prefractured and granular $\mathrm{Al}_{2} \mathrm{O}_{3}[61,62]$ and $\mathrm{SiC}[63,64]$. The micromechanism is dependent on particle size, and two principal mechanisms were identified:

1. Large particles: when the particles are larger than the critical flaw size for fracture, under imposed conditions of stress and strain rate, they fracture. The comminution (or attrition, as called by Potapov and Campbell [65]) is an integral part of the localization process. Particles break and this process produces a localization region. Particles adjoining the localization region are 'dragged' into the shear band and undergo erosion. The fine particulates formed in the shear band act as a lubricant and ensure localization. Fig. 12(a) and (b) show this process for prefractured and granular $\mathrm{SiC}$; a schematic representation is shown in Fig. 12(c) [63,64].

2. Small particles: when the size of particles is below $a_{\mathrm{c}}$, the critical flaw size, they no longer fracture under the imposed conditions. Shih et al. $[63,64]$ calculated the critical particle size for the change in regime. They used a Weibull distribution of flaws and a Griffith failure criterion. Fig. 13 shows the resulting analytical prediction. It is found that failure is initiated in the region of the sphere (idealized shape) in which the stress state (i.e. three principal stresses) is compressive. The critical particle size (particle size below which no fracture occurs, at the prescribed superimposed pressure) decreases as the superimposed stress is increased. Three critical particle sizes are shown in Fig. 13: $a_{\mathrm{c} 1}, a_{\mathrm{c} 2}$, and $a_{\mathrm{c} 3}$. They correspond to different threshold values of the superimposed pressure: $P=5$ GPa for $a_{\mathrm{c} 1}=0.4 \mu \mathrm{m} ; P=2 \mathrm{GPa}$ for $a_{\mathrm{c} 2}=4 \mu \mathrm{m}$; and $P=1 \mathrm{GPa}$ for $a_{\mathrm{c} 3}=40 \mu \mathrm{m}$. As the superimposed pressure decreases, the critical particle size increases. Since there is no comminution within the shear localization region when the particle size is below the critical size for fracture, the particles flow past each other and undergo plastic deformation and repacking. This results in significant heat evolution, and temperature rises that lead to fusing of the particles and bonding. The calculated temperature rise for $\mathrm{SiC}$, assuming a bilinear strength dependence of temperature, is shown in Fig. 14. It was obtained from the classic Fourier heat transfer equation:

$\frac{\partial}{\partial t}(\rho C T)=k_{1} \frac{\partial^{2} T}{\partial x^{2}}+\beta \tau \frac{\partial \gamma}{\partial t}$

where $k_{1}$ is the heat conductivity, $\beta$ is a coefficient describing the efficiency of converting plastic work into heat; $\rho$ and $C$ are the material density and specific heat. The temperature in the center of the band rises to $2400^{\circ} \mathrm{C}$, for a shear strain of 12 and $\beta=1$. Fig. 15 shows the appearance of the shear band for $\mathrm{Al}_{2} \mathrm{O}_{3}$ (Fig.15(a)) and $\mathrm{SiC}$ (Fig. 15(b)). In the alumina (unpol- 


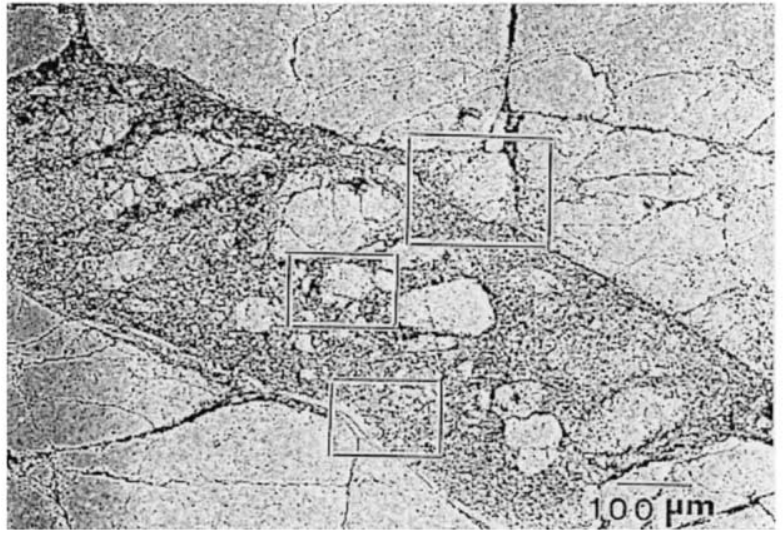

(a)

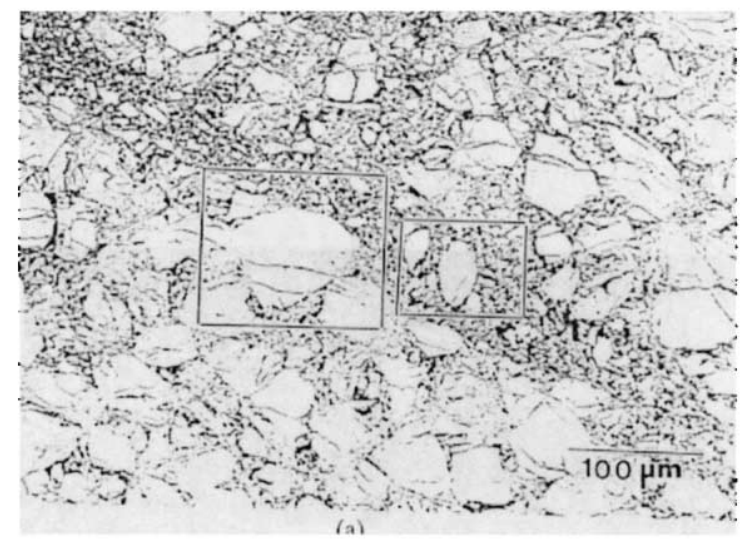

(b)

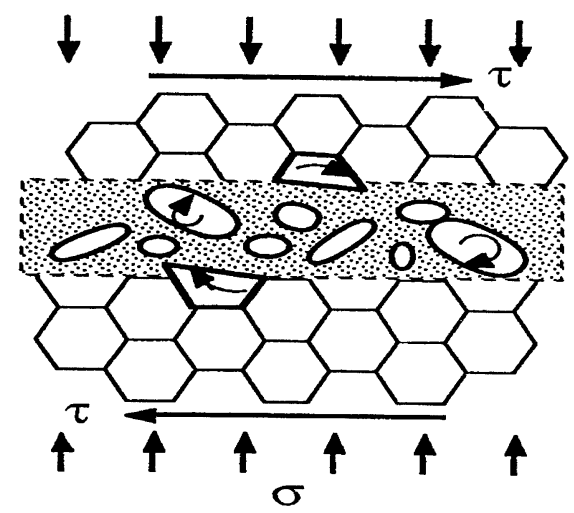

(c)

Fig. 12. Micromechanism of shear band formation in (a)prefractured; (b)granular ceramics. The schematic representation of mechanism is shown in (c)

ished section shown), the particles are deformed and show evidence of plastic flow (see arrows). In the SiC (polished section shown), complete bonding has occurred, and the microhardness indentation revealed the same value (26 GPa) as for bulk, fully densified material. The original particles cannot any longer be seen in the polished section of SiC. This is clear and irrefutable evidence for plastic deformation and heating.

\subsection{Non-crystalline materials}

The absence of work hardening of many non-crystalline materials (polymers and glassy metals) predisposes them to shear localization. The mechanisms by which inelastic deformation takes place have been postulated by Deng et al. [6] and Bulatov and Argon [7-9] and involve the nucleation of shear ellipsoids, in which the atoms undergo reorganization and plastic strain ensues. It has also been shown by Bulatov and Argon [9] that these strain ellipsoids organize themselves into shear bands. The experiments carried out by Pershin and Nesterenko [67] for metallic glass show that, under dynamic loading conditions, plastic deformation is concentrated into shear bands. The metallic glass foil (30 $\mu \mathrm{m}$ thick), $\mathrm{Co}_{58} \mathrm{Ni}_{10} \mathrm{Fe}_{5} \mathrm{Si}_{11} \mathrm{~B}_{16}$, was wrapped into a cylindrical configuration and submitted to a slightly modified version of the explosive loading geometry presented in Fig. 2(b). The displacements within the shear bands as well as the thickness can be readily seen from the markings. Fig. 16 shows a typical shear band, evident from the offset on the foil. The shear band thickness was characteristically $5 \mu \mathrm{m}$ for this alloy, and shear strains in the range of $4-8$ were observed. Not much can be said, at present, about the microstructural evolution within the shear bands, but it can be speculated that it remains glassy. The shear band thickness is considerably below that of the foil, ensuring a cooling rate that is high. Nevertheless, the possibility of crystallization cannot be excluded, and transmission electron microscopy can reveal the microstructural details.

\section{Self organization}

\subsection{Trajectories and spacing}

All experiments carried out using the thick walled cylinder methods revealed that the shear bands do not occur in a random manner, but with a well established and self-organized pattern. Their trajectories were, in all cases, spiral.

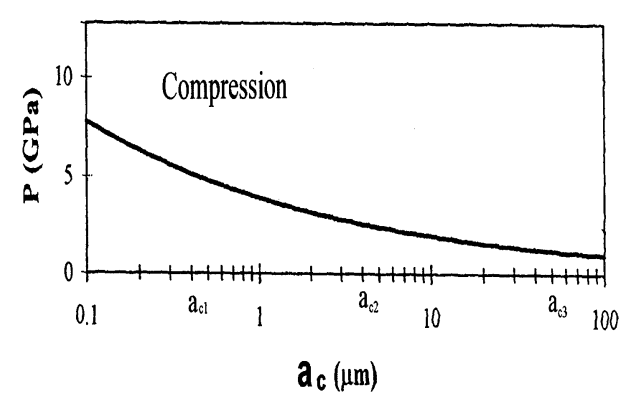

Fig. 13. Relationship between external pressure $\mathrm{P}$ and critical particle size for comminution 


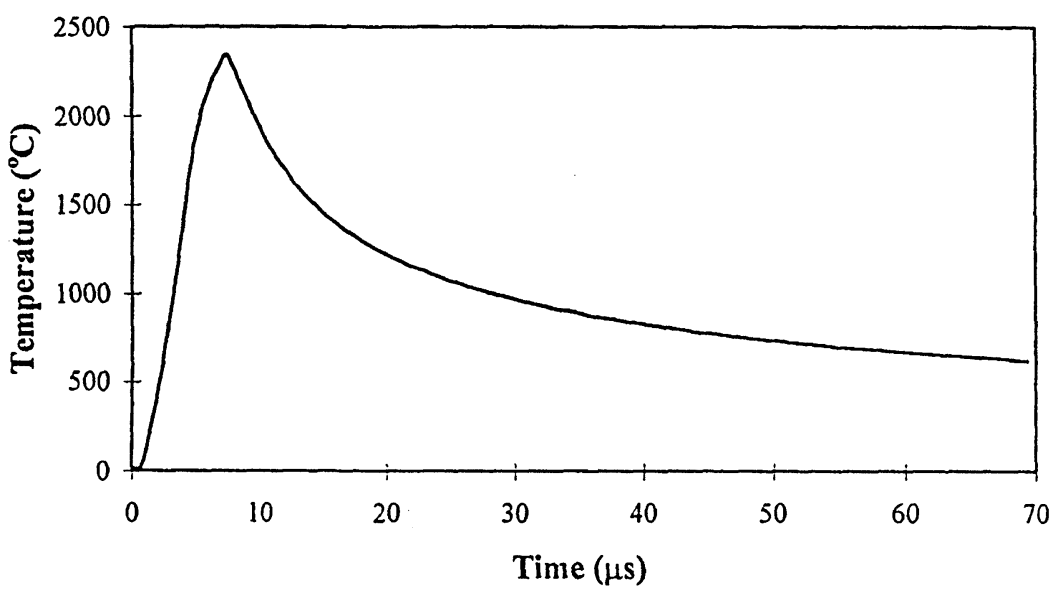

Fig. 14. Calculation temperature at the middle of $\mathrm{SiC}$ shear band (shear band width: $25 \mu \mathrm{m} ; \gamma_{\mathrm{s}}=12$ ) (from Shih et al.[64]).

The shear band morphology can be obtained by calculating the trajectory of the tip of the shear localization region. Fig. 17(a)-(c) show, in a schematic fashion, the initiation and propagation of shear bands. The following assumptions can be made:

1. The overall movement of the material, at distances relatively far outside the central hole, is radially convergent with an axis of symmetry coincident with the axis of the cylinder. In actual deformation, the geometrical constraints are such that an additional bending of the shear segments is required for the total collapse of the void.

2. The critical effective strains for shear band initiation (at radius $R_{\mathrm{i}}$ ) and propagation are identical and independent of strain rate. Under pure shear, the hypothesis of critical effective strain or critical shear strain are identical.

3. Shear-band initiation takes place at the internal surface of the hole and all shear bands are created simultaneously.

4. The tip of a shear band propagates along the surfaces of maximum shear strain.

5. Material is incompressible.

The critical shear strain for shear-band initiation is equal to (see Fig. 17):

$\gamma_{\mathrm{c}} \approx 2 \ln \left(\frac{R_{0}}{R_{\mathrm{i}}}\right)=\ln \left(\frac{R_{0}^{2}}{r_{\mathrm{f}}^{2}}+1\right)$

$r_{\mathrm{f}}$ is the final radius of the shear-band extremity; $R_{0}$ and $R_{\mathrm{i}}$ are given in Fig. 17. The propagating shear band at an intermediate stage of the collapse process is depicted in Fig. 17(d), an element undergoes a displacement from point 1 (at radius $r_{1}$ ) to point 2 (at radius $r$ ) after complete collapse. Point 3 indicates in a schematic fashion the break in symmetry produced by a shear band. The shear band forms at an angle of $45^{\circ}$ with the radius at the point $r_{1}$. This angle will decrease as deformation proceeds; it is possible to establish the change in $\alpha$ with $r$.
With the assumption (Assumption 1) that the material with shear bands maintains its movement close to cylindrical symmetry, the radial and hoop true strains from the point corresponding to the initial radius $r_{1}$
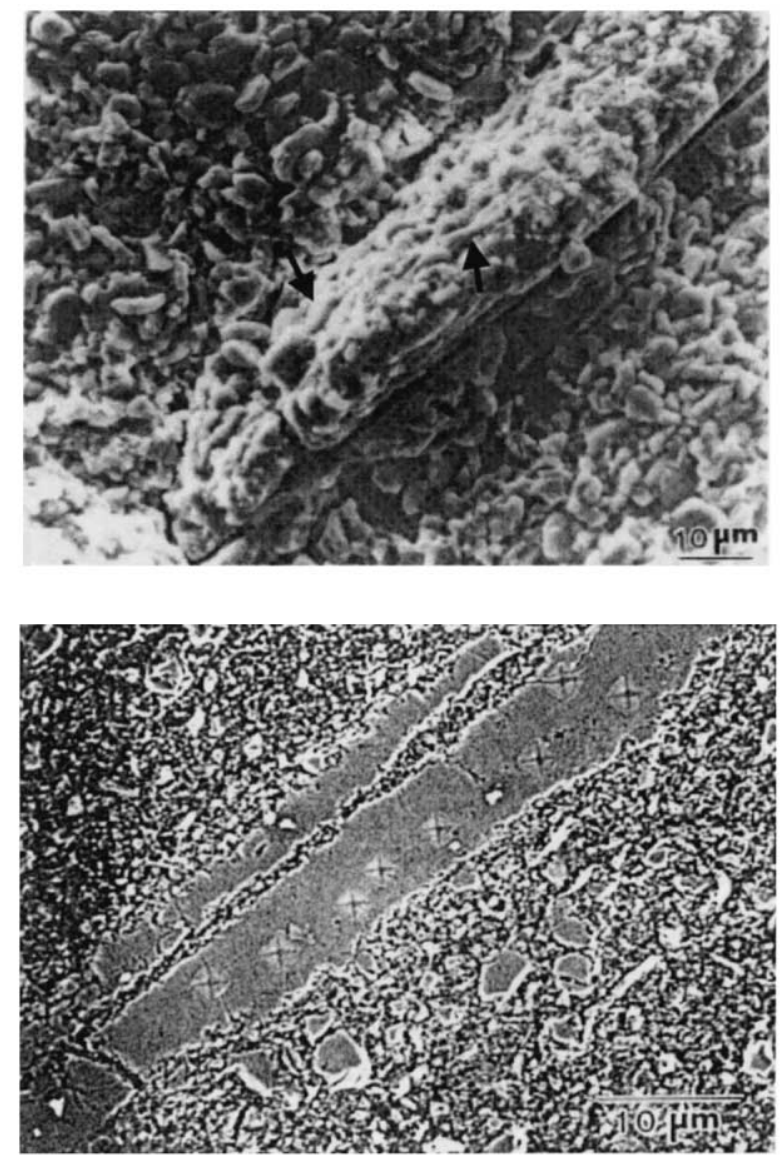

Fig. 15. (a) Shear band in $\mathrm{Al}_{2} \mathrm{O}_{3}$ (SEM); notice deformed particles and effect of heat(from Nesterenko et al.[71]). (b) shear band in SiC (polished surface; SEM), Notice hardness indentation at center of the band(from Shih et al.[64]). 


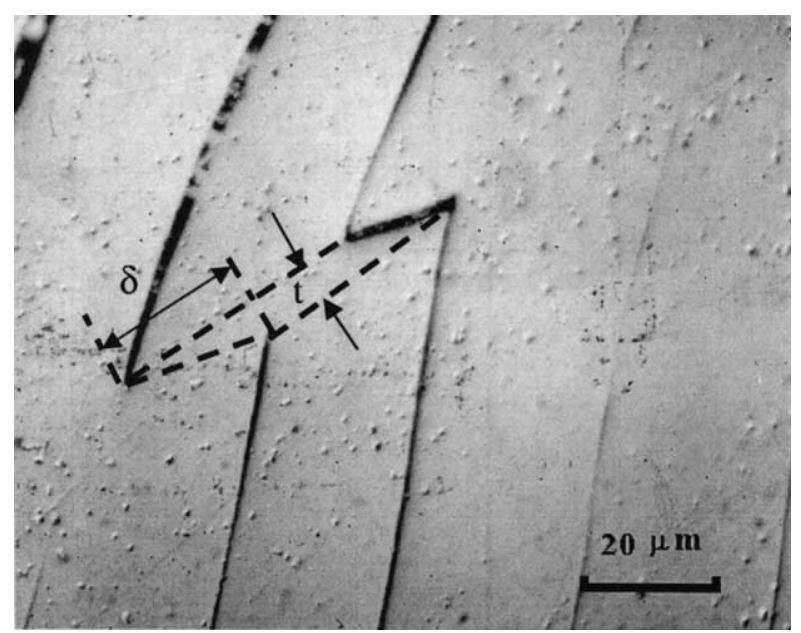

Fig. 16. Shear band in $\mathrm{Co}_{58} \mathrm{Ni}_{10} \mathrm{Fe}_{5} \mathrm{Si}_{11} \mathrm{~B}_{16}$ glass; displacement $\delta$ and shear-band thickness $\mathrm{t}$ are marked in photograph.

(the position corresponding to shear band creation), up to the end of the collapse process $\left(r_{1} \rightarrow r\right)$, are:

$\varepsilon_{r r}=\ln \left(\frac{r_{1}}{r}\right), \quad \varepsilon_{\varphi \varphi}=\ln \left(\frac{r}{r_{1}}\right)$

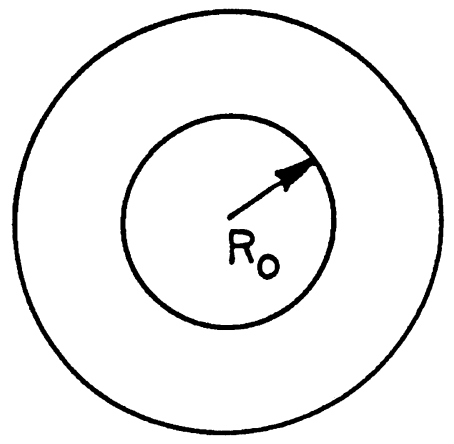

(a)

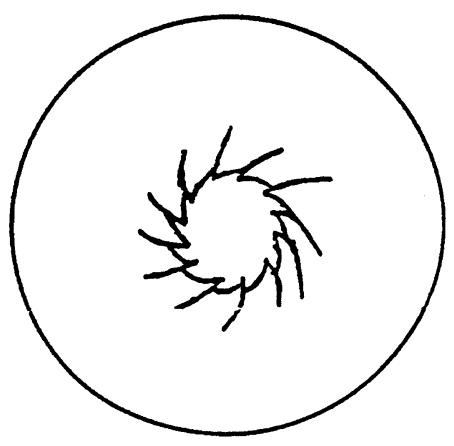

(c)
Using Eq. (39) it is possible to obtain an equation for the angle $\alpha$ of the shear band with the radial direction (Fig. 17(d)):

$\tan \alpha=\mathrm{e}^{-2 \varepsilon_{r r}}=\left(\frac{r}{r_{1}}\right)^{2}$

The shear strain at $r_{1}$, according to Assumption 2, should be equal to $\gamma_{c}$; this leads to an expression for $r_{1}$ :

$2 \ln \left(\frac{r_{10}}{r_{1}}\right)=\gamma_{\mathrm{c}} \Rightarrow r_{1}=r_{10} \mathrm{e}^{-\gamma_{\mathrm{c}} / 2}$

The radius $r_{10}$ corresponds to the initial position of the point that has radius $r_{1}$ when the shear band starts. Combining Eqs. (40) and (41) and using the relation $r_{10}^{2}-R_{0}^{2}=r^{2}$ (constancy of volume), one obtains an expression for $\alpha$ in a fully collapsed cylinder in terms of the initial hole radius $R_{0}$, the radius of the final point of the shear band extremity $r_{\mathrm{f}}$, and a general radius $r$ :

$\tan \alpha=\frac{r^{2}}{r_{\mathrm{f}}^{2}} \frac{\left(R_{0}^{2}+r_{\mathrm{f}}^{2}\right)}{\left(R_{0}^{2}+r^{2}\right)}$

It is easy to see that when $r$ approaches zero, the

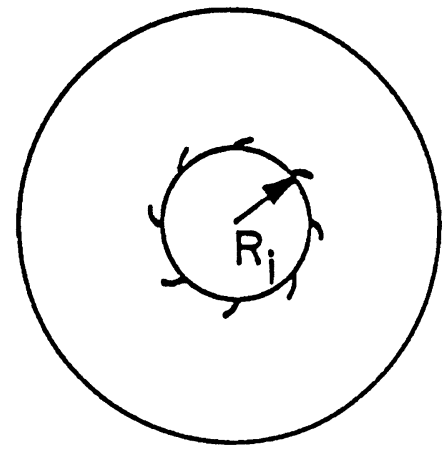

(b)

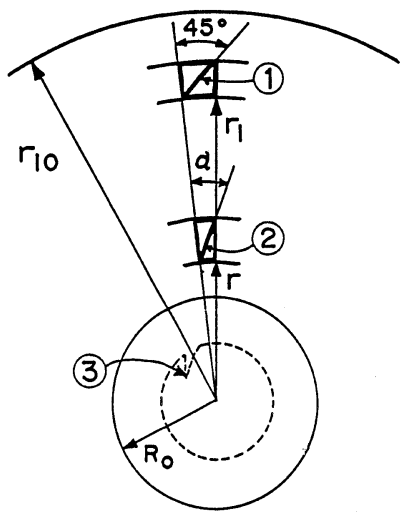

(d)

Fig. 17. (a-c) Generation and propagation of shear bands in collapse of thick-walled cylinder; (d) Deformation of a material element with an initiating shear band as it is displaced from original position at a general radius $r\left(\alpha<45^{\circ}\right)$. 


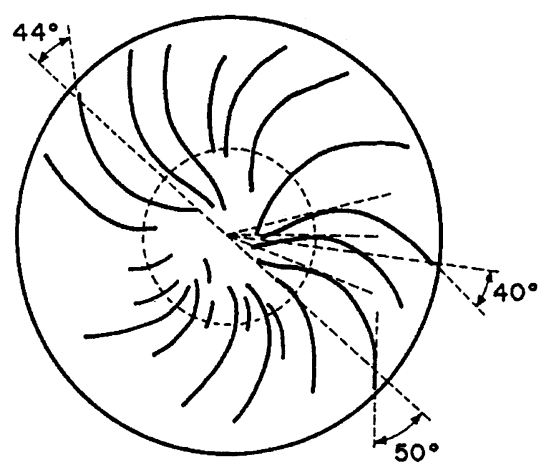

(a) experimental tracing for titanium

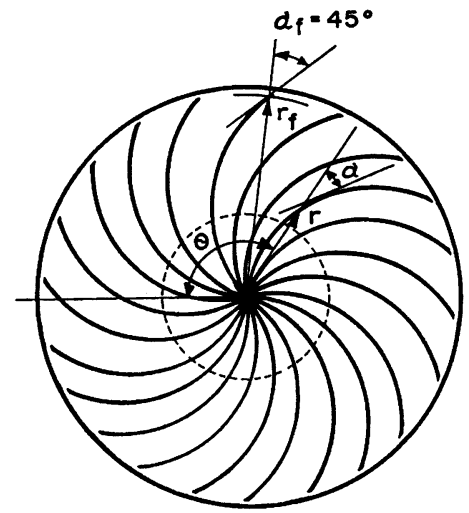

(b) calculated pattern

Fig. 18. Shear-band patterns on cross-section produced by collapse of thick-walled cylinder; (a) experimental tracing for titanium; (b) calculated pattern

angle $\alpha \rightarrow 0$, providing a more radial-like geometry of shear bands in the vicinity of collapsed hole.

Eq. (41) enables one to find the geometry of the shear band after complete pore collapse in closed form. In polar coordinates $r, \theta$ :

$\tan \alpha=r \frac{\mathrm{d} \theta}{\mathrm{d} r}$

By combining Eqs. (42) and (43) one obtains an equation for the shear band in a completely collapsed cylinder in the frame of the aforementioned assumptions:

$\theta=\frac{\left(R_{0}^{2}+r_{\mathrm{f}}^{2}\right)}{r_{\mathrm{f}}^{2}} \int_{0}^{r} \frac{r \mathrm{~d} r}{\left(R_{0}^{2}+r^{2}\right)}=\frac{1}{2}\left(1+\frac{R_{0}^{2}}{r_{\mathrm{f}}^{2}}\right) \ln \left(1+\frac{r^{2}}{R_{0}^{2}}\right)$

Eq. (44) predicts the general morphology of a shear band. An equation can be developed that describes the entire assemblage of shear bands by assuming that they create a periodical array with spacing $L$ and the same sense. In order to do this in a more general way, the critical shear stress for shear band initiation is used to replace $r_{\mathrm{f}}$; thus, the solution acquires generality. The number of shear bands forming on the circumference has to be an integer, and therefore the calculated number $i$ has to have an integral upper bound, which is indicated by 'int' below. Each solution $i$ represents one curve.

$\theta_{i}=\mathrm{e}^{\gamma_{\mathrm{c}} / 2}\left\{\frac{i \sqrt{2} L}{R_{0}}+\frac{\mathrm{e}^{\gamma_{\mathrm{c}} / 2}}{2} \ln \left(1+\frac{r^{2}}{R_{0}^{2}}\right)\right\}$

$\operatorname{int}\left(\frac{2 \pi R_{0}}{\sqrt{2} L} e^{-\gamma_{\mathrm{c}} / 2}\right) \geq i \geq 1$

This expression was derived with the basic assumption that the tip of the shear band (outermost extremity, which propagates outwards) is subjected to growth governed by the orientation of maximum shear stress
( $45^{\circ}$ with the radial direction). The shear band left behind is assumed to homogeneously move inward with tube collapse. Shear-band trajectories produced by the complete collapse of a titanium cylinder are shown in Fig. 18(a) and compared with the prediction in Fig. 18(b). The good agreement obtained is clear. In the calculated trajectories, the number $N$ was set equal to 20, based on the experiments. All shear bands were clockwise spirals in this particular experiment [66].

Cross-sections of experiments carried out for a number of materials representative of metals (Ti, 304SS), ceramics $\left(\mathrm{Al}_{2} \mathrm{O}_{3}\right.$ and $\left.\mathrm{SiC}\right)$, and polymers (teflon) are shown in Fig. 19. In all experiments the characteristic logarithmic spiral configuration described in Fig. 18 is observed. Since the collapse is only partial for all experiments except for teflon, the spirals do not converge completely towards the center. Nevertheless, the periodic nature of the shear bands is evident, and they tend to group themselves into regions of clockwise and regions of counterclockwise rotation. In some cases only one family (clockwise or counterclockwise) is observed; for most cases, two or four families are observed. The measurement of the spacing along the internal wall of the cylinder, corrected for the fact that the bands form at $45^{\circ}$ from the radial direction $(\sqrt{2} / 2)$ provides the spacing. This spacing is given in Table 3 and is a function of a number of parameters. This research is only at its early stages, but distinct differences can be observed. The spacings range from 0.1 to $3.2 \mathrm{~mm}$.

For the metallic glass, a slightly different experimental configuration was used [67]. Nevertheless, a characteristic shear-band spacing was also revealed, and is clearly shown in Fig. 20. Four shear bands are shown by arrows. The mean spacing between shear bands was found to be $0.6 \mathrm{~mm}$, consistently with titanium and teflon. 


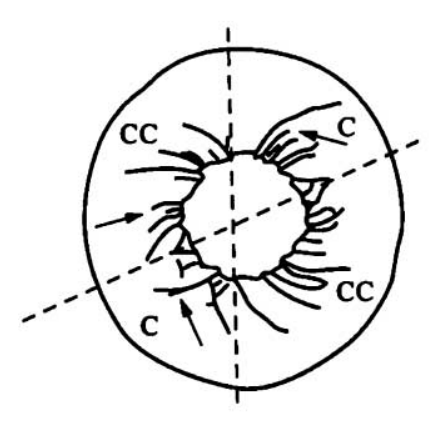

(a) Titanium

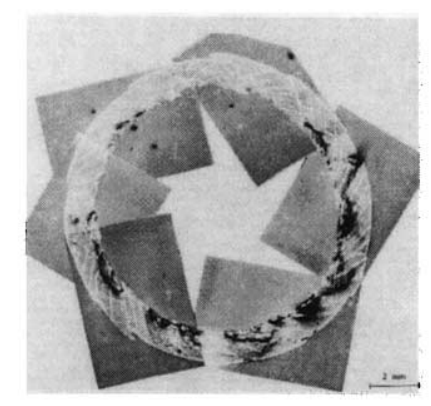

(c)Granular $\mathrm{Al}_{2} \mathrm{O}_{3}$

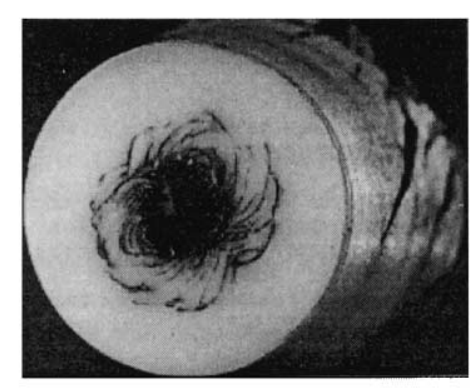

(e)Teflon

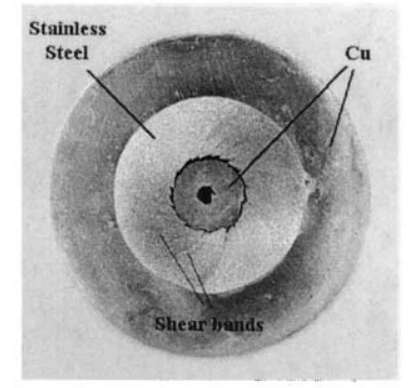

(b) Stainless steel 304

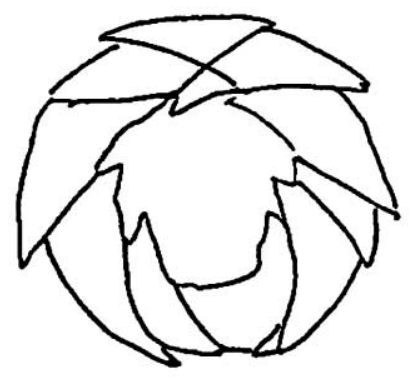

(d) Prefractured Si C

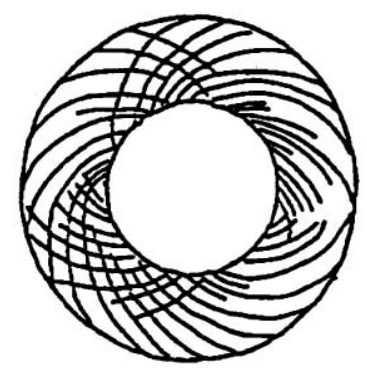

(f) Granular SiC

Fig. 19. Cross-section of thick-walled cylinder subjected to partial collapse with different materials showing similar pattern of shear bands.

\subsection{Metals}

The current models for predicting the shear band spacing are based on different analyses of shear banding. The basic equations for shear localization with shear along direction Ox axis are the momentum and energy conservation equations including thermal transfer:

$\rho \frac{\partial v}{\partial t}=\frac{\partial \tau}{\partial y}$

$\rho C \frac{\partial T}{\partial t}=\beta \tau \dot{\gamma}+k_{1} \frac{\partial^{2} T}{\partial y^{2}}$

where $v$ is material velocity, and $\tau$ and $\dot{\gamma}$ are shear stress and shear strain rate, respectively. The other symbols were defined previously. $\beta$ is the thermal conversion coefficient. This factor is usually in the $0.9-1$ range.
Eq. (48) is identical to Eq. (37); the conversion factor $\beta$ has, however, a different interpretation, because fracture is a major process in ceramics.

Table 3

Shear Band Spacing

\begin{tabular}{lll}
\hline Materials & Characteristics & Spacing, mm \\
\hline CP Titanium & Initial & 3.15 \\
& Well-developed & 0.6 \\
Stainless Steel & Initial & 0.12 \\
$\mathrm{SS} \mathrm{304L}$ & Well-developed & 3.2 \\
$\mathrm{Al}_{2} \mathrm{O}_{3}$ & Granular & $0.79-1.04$ \\
$\mathrm{SiC}$ & Prefractured & 1.8 \\
& Granular & $0.4-1$ \\
$\mathrm{Teflon}$ & Prefractured & $1.7-3$ \\
${\mathrm{Metallic} \mathrm{Glass}}_{\mathrm{Co}} \mathrm{Ni}_{10} \mathrm{Fe}_{5} \mathrm{Si}_{11} \mathrm{~B}_{16}$ & & 0.6 \\
\hline
\end{tabular}




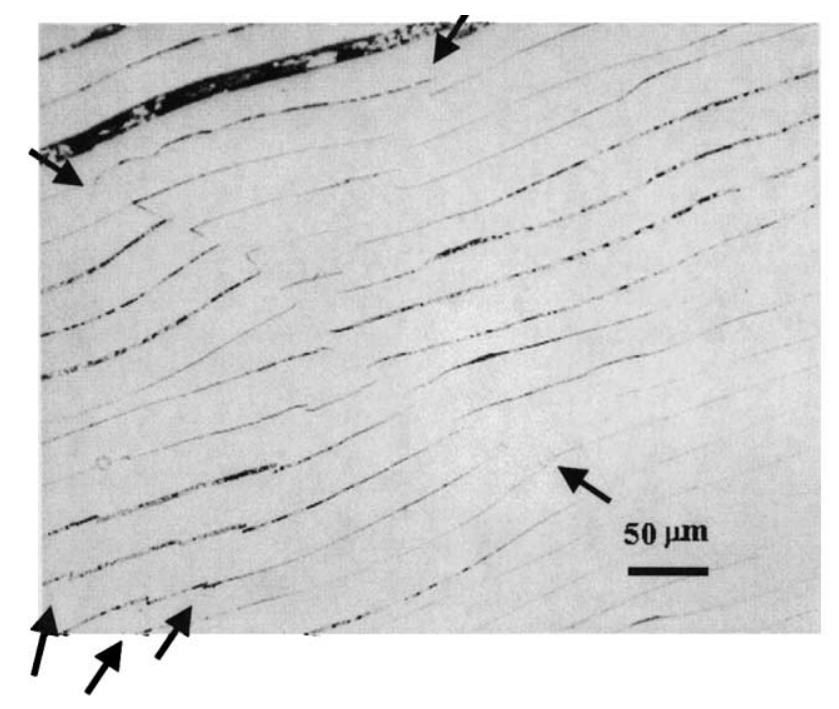

Fig. 20. Shear bands in metallic glass in cylindrical collapse geometry (arrows indicate shear bands)

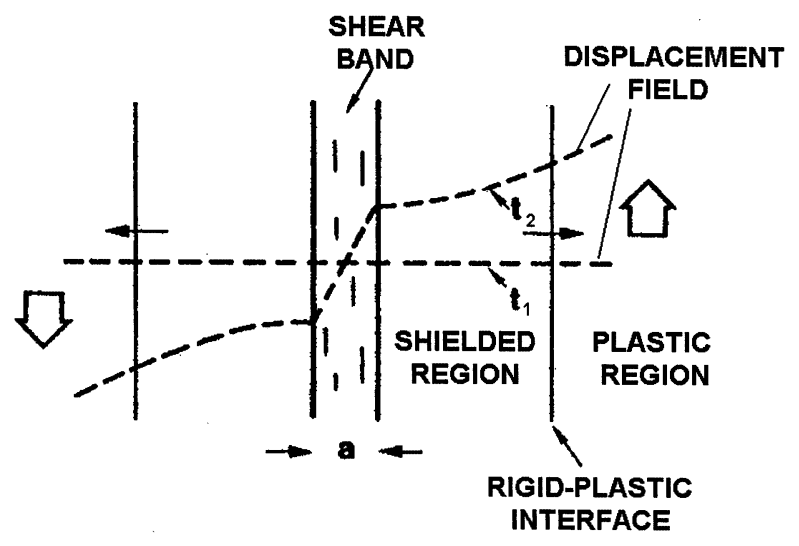

(a)

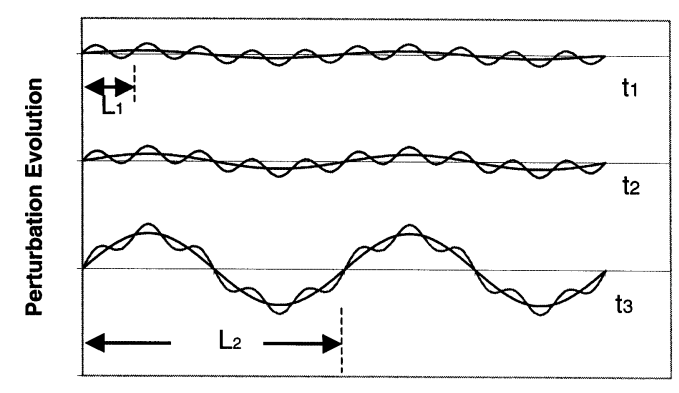

(b)

Fig. 21. Two mechanisms of determining shear band spacing (a) momentum diffusion(Adapted from Grady and Kipp[68]); and (b) growth of perturbation

Three possible mechanisms for the determination of shear band spacing are discussed below: (a) the GradyKipp momentum diffusion mechanism; (b) the WrightOckendon-Molinari perturbation mechanism; (c) a mechanism based on microstructural inhomogeneities.
The first theoretical model for shear band spacing was proposed by Grady and Kipp [68]. In this approach, the rapid loss of strength across the developing shear band affects neighboring material by forcing it to unload. This process is communicated outward by momentum diffusion, rather than by elastic wave propagation. Fig. 21(a) shows a shear band (thickness $a$ ) with the displacement fields at two times $t_{1}$ and $t_{2}$. At $t_{1}$ the displacement is zero. A shielded region propagates away from the band. It is represented by the rigid-plastic interface in Fig. 21(a). The minimum separation between independently nucleating bands arises from computing the distance traveled by the diffusive unloading front during the time required to unload as localization occurs. The predicted spacing, $L_{\mathrm{GK}}$ is:

$L_{\mathrm{GK}}=2\left[\frac{9 k_{1} C}{\dot{\gamma}_{0}^{3} a^{2} \tau_{0}}\right]^{1 / 4}$

In Eq. (49) the applied shear strain rate is $\dot{\gamma}_{0}$, and the flow stress is assumed to be linear function of temperature with a softening coefficient $a\left(T_{\mathrm{r}}\right.$ is a reference temperature, at which the flow stress is equal to $\left.\tau_{0}\right)$ :

$$
\tau=\tau_{0}\left[1-a\left(T-T_{\mathrm{r}}\right)\right]
$$

Grady and Kipp (GK) [68] considered a simple rateindependent material. Wright and Ockendon (WO) [69] developed an analysis based on the notion that shear bands arise from small growing disturbances in an otherwise uniform region. This concept had been previously advanced by Grady [70]. Disturbances do not propagate in perpendicular directions, but simply grow in place. Fig. 21(b) shows, in schematic fashion, two wave trains at successive times $t_{1}, t_{2}$, and $t_{3}$. The wave with the largest wavelength, $L_{2}$, shows a higher rate of amplitude increase. Thus, it gradually dominates the other wavelength, and will determine the spacing of the shear bands. The most likely minimum spacing is obtained by finding the fastest growing wavelength.

Wright and Ockendon [69] applied the perturbation analysis to the conservation equations according to the approach introduced by Clifton [72]. The three equations and obtained a spacing, $L_{\mathrm{wO}}$, equal to:

$L_{\mathrm{wO}}=2 \pi\left[\frac{m^{3} k_{1} C}{\dot{\gamma}_{0}^{3} a^{2} \tau_{0}}\right]^{1 / 4}$

The material flow stress was assumed to be both temperature and strain-rate dependent; hence Eq. (50) was modified by incorporating strain rate effects through parameter $m$ ( $\gamma_{0}$ is a reference strain rate):

$\tau=\tau_{0}\left[1-a\left(T-T_{0}\right)\right]\left(\frac{\dot{\gamma}}{\dot{\gamma}_{0}}\right)^{m}$

Although the approaches taken by GK and WO are completely different, the former concentrating on the stress collapse mechanism, and the latter concentrating on the earliest stages of localization, it is a remarkable 


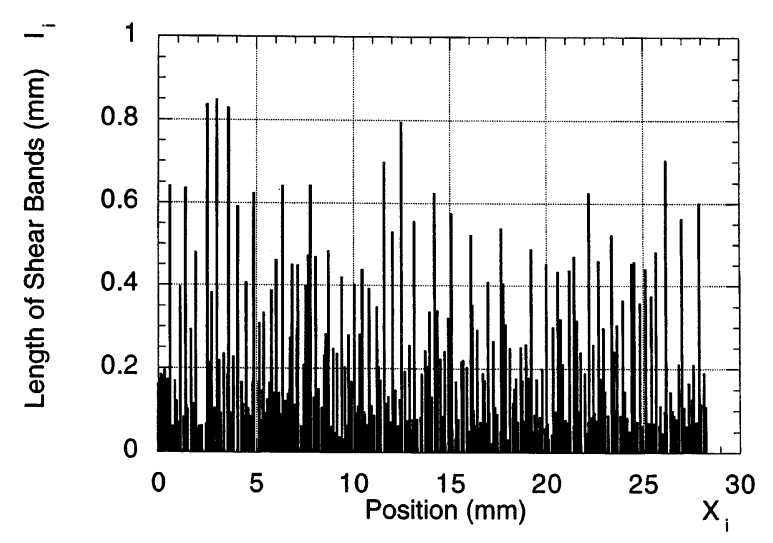

(a) Initial stage

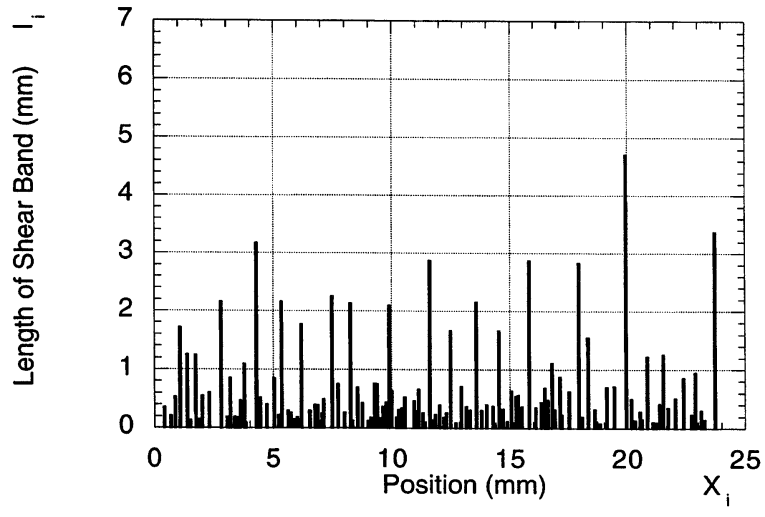

(b) Developed stage

Fig. 22. Pattern of shear bands in stainless steel at different stages: (a) initial stage, $\varepsilon_{\text {eff }}=0.541$; (b) shear bands propagate and the new spacing is developing, $\varepsilon_{\mathrm{eff}}=0.915$.

fact that except for numerical factors and the rate constants, the two results are the same. The major difference between Eqs. (49) and (51) is that strain rate sensitivity affects the spacing in WO model and is not present in the GK model.

Molinari [71] further developed the analysis; in this model the important step which determines the shear band spacing is related to the early stages of flow localization, as in the WO model. The important feature of Molinari's model is that it includes the strainhardening effect; the non-strain-hardening case can be obtained as a special case. For power law strain hardening, Molinari [71] obtained the spacing, given by
$L_{\mathrm{M}}=\frac{2 \pi}{\xi_{0}}\left(1+\frac{3}{4} \frac{\rho C \frac{\partial \dot{\gamma}}{\partial \gamma}}{\beta \tau_{0} \frac{\partial \dot{\gamma}}{\partial T}}\right)$

for a constitutive equation of the form:

$\tau=\mu_{0}\left(\gamma+\gamma_{i}\right)^{n}\left(\frac{\dot{\gamma}}{\dot{\gamma}_{0}}\right)^{m}\left(\frac{T}{T_{\mathrm{r}}}\right)^{v}$

where $\gamma_{i}$ is a pre-strain and $\xi_{0}$ is the wave number of the perturbation; $v, n, m$ and $\mu_{0}$ are the material parameters. When the work hardening is absent $(n=0)$, Eq. (53) reduces itself to:

$L_{\mathrm{M}}=2 \pi\left[\frac{m^{2} k_{1} C\left(1-a T_{0}\right)^{2}}{\left(1-m^{-1}\right) \beta^{2} \dot{\gamma}_{0}^{3} \tau_{0} a^{2}}\right]$

This equation is similar to WO except for the term $\left(1-a T_{0}\right)^{2}$ which can create a difference in calculated values on the order of 0.3 .

Nesterenko et al. [73] carried out experiments on titanium in which the maximum strain was varied by selecting the appropriate stopper tubes(see Fig. 2(b) and (c)). A detailed analysis and characterization was also carried out for AISI 304L stainless steel, subjected to two levels of plastic strain in the TWC method [74]. At an initial stage of plastic deformation $\left(\varepsilon_{\mathrm{eff}}=0.541\right)$, a large number of small shear bands with lengths varying between 0.1 and $0.8 \mathrm{~mm}$ were obtained (Fig. 22(a)). The characteristic spacing was $0.12 \mathrm{~mm}$. At an increased level of plastic deformation $\left(\varepsilon_{\text {eff }}=0.915\right)$ the number of the small bands $(<0.8 \mathrm{~mm}$ length) remained essentially the same, while the spacing between the larger shear bands had considerably increased, to $3.2 \mathrm{~mm}$ (Fig. 22(b)). Predictions using Eqs. (49), (51) and (55), for $\mathrm{G}-\mathrm{K}, \mathrm{W}-\mathrm{O}$, and Molinari models, respectively, are also shown in Table 4. It is clear that the WO-M mechanism describes the initiation better, whereas GK represents the shear-band spacing at a well developed stage. These results are only preliminary; nevertheless, they indicate that both mechanisms might be operating. Similar results were obtained by Nesterenko et al. [73] for titanium: the perturbation analysis represents the initiation stage better, whereas propagation is affected by the momentum diffusion equation. Prior to the onset of localization, momentum diffusion is absent, and its role is only felt in the propagation stage. Local fluctuations in strain and temperature lead to the initiation. Additionally, the surface of the inner cylinder wall contains imperfections which lead to perturbations.

Table 4

Theoretical predictions and the experimental results for stainless steel

\begin{tabular}{llllll}
\hline SS 304L & Exp. Data Initial stage & Late stage Exp. Data & $\mathrm{L}_{\mathrm{Wo}}(\mathrm{mm})$ & $\mathrm{L}_{\mathrm{GK}}(\mathrm{mm})$ & $\mathrm{L}_{\mathrm{MO}}(\mathrm{mm})$ \\
\hline Spacing $(\mathrm{mm})$ & 0.117 & 3.199 & 0.166 & 2.62 & 0.160 \\
\hline
\end{tabular}




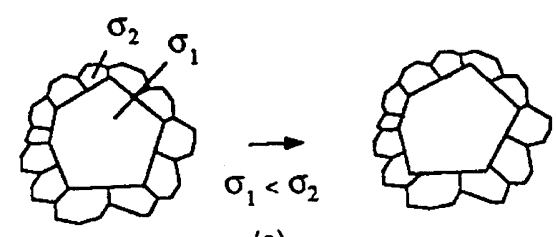

(a)

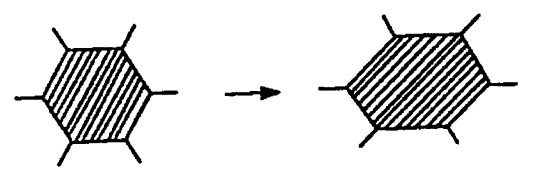

(b)
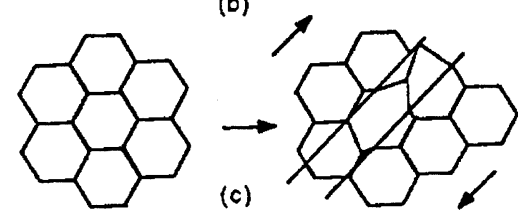

(c)
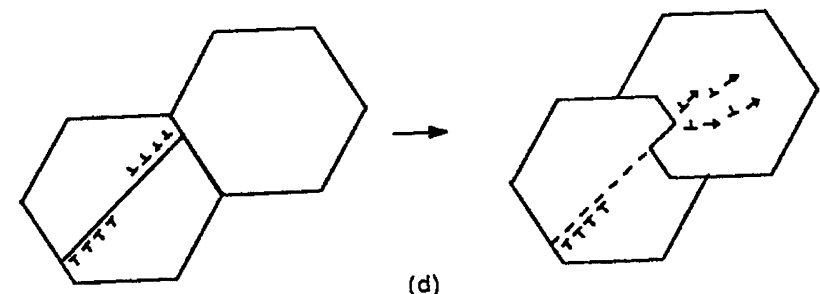

(d)

Fig. 23. Shear band initiation by microstructural inhomogeneities: (a) grain-size inhomogeneity; (b) geometrical softening; (c) Peirce-Asaro-Needleman textural localization and (d) dislocation pile-up release (from Nesterenko et al.[73]).

It is important to connect the $\mathrm{WO}-\mathrm{M}$ and $\mathrm{GK}$ models to microstructural processes happening in the material. It is even possible that microstructural inhomogeneities rather than strain/temperature perturbations or momentum diffusion, are the determining factors in spacing. Possible micromechanisms for the initiation of shear bands have been discussed by Meyers et al. [16] and Nesterenko et al. [73]. A few possible microstructural initiation sites are shown in Fig. 23; they are all at grain scale. They are briefly discussed below. There is always a distribution of grain sizes within a material, and larger grains exhibit a lower yield stress $\left(\sigma_{1}\right.$, in Fig. 23), whereas smaller grains have a higher yield stress $\left(\sigma_{2}\right.$ in Fig. 23). The large grain will deform preferentially and could be an initiation site. Grain rotation can lead to softening, which is shown schematically in Fig. 23(b). The increase in Schmid factor of a grain with plastic deformation leads to localized softening which can initiate a shear band. The localized deformation of one grain can propagate along a band as shown in Fig. 23(c). This mechanism of localization through the cooperative plastic deformation of grains has been modeled by Peirce et al. [74], and Anand and Kalidindi [75]. A fourth mechanism is shown in Fig. 23(d). A dislocation pile-up, upon bursting through a grain boundary, can generate the local temperature rise and plastic deformation that would initiate shear band. This mechanism has been proposed by Armstrong and Zerilli [76].

\subsection{Ceramics and glasses}

In ceramics, there is a distinct difference between prefractured and granular material. $\mathrm{Al}_{2} \mathrm{O}_{3}$ and $\mathrm{SiC}$ showed consistent shear band spacings (Table 3): 0.4-1 $\mathrm{mm}$ for the granular condition and $1.7-3 \mathrm{~mm}$ for the prefractured condition. The reasons for these differences are still poorly understood and could be due to the softening rate. In the momentum diffusion formulation, the higher the unloading rate along a band, the lower the spacing among shear bands. Differences in initial density could also account for the greater shearband spacing for the pre-fractured material: the density of the (pre-compacted) granular ceramic is only $85 \%$ of the theoretical value.

For metallic glasses and polymers practically nothing is known; the spacing seems to be around $0.6 \mathrm{~mm}$.

\section{Conclusions}

Shear localization is known to play an important role in the inelastic deformation of materials at high strain rates. The microstructural processes occurring in the different classes of materials are presented:

1. Metals: Thermal softening is the first stage of this process, leading to processes of dynamic recovery and recrystallization with associated drops in the flow stress. Calculations reveal, for the first time, that the break-up of the elongated sub-grains and diffusive rotation of the grain boundaries can occur during the deformation process. This is direct support for the mechanism of rotational dynamic recrystallization proposed by Meyers and Pak [25] and Meyers et al. [16] for titanium, Andrade et al. [14] for copper, and Nesterenko et al. [73] for tantalum.

2. Ceramics and rocks: Granular and prefractured ceramic $\left(\mathrm{Al}_{2} \mathrm{O}_{3}\right.$ and $\left.\mathrm{SiC}\right)$ were analyzed and shear localization was found to occur by two different mechanisms, dependent upon particle size:

2.1. particle break-up/comminution, when the size exceeds a critical value $a_{\mathrm{c}}$.

2.2. Particle repacking and plastic deformation with significant heat evolution leading, under extreme conditions, to resintering of particles when size is below the critical value $a_{\mathrm{c}}$.

3. Non-crystalline metals and polymers: The deformation processes leading to large scale localization are most probably initiated by Argon 'local inelastic transformations' that form in a cooperative manner and organize themselves into shear zones. Further investigation of this phenomenon by advanced char- 
acterization methods is necessary to elucidate the mechanisms.

These shear bands constitute the primary carriers of inelastic deformation in high-strain rate deformation. In a manner analogous to dislocations, that are the primary carriers of plastic deformation in metals, the shear bands self-organize themselves. Their spacing, which is dependent on material and loading parameters, was investigated in a collapsing thick-walled cylinder geometry. The initiation of shear localization suggests a spacing dictated by the following mechanisms:

1. Perturbation of the plastic deformation field with spacing dictated by wavelength of perturbation showing highest growth rate.

2. Momentum diffusion, through the unloading of material surrounding a shear band, creating a shielded region in which further shear localization is inhibited.

3. Microstructural inhomogeneities determining the initiation sites and, consequently, spacing of bands.

\section{Acknowledgements}

Research funded by the US Army Research Office(Contract DAAH 04-96-1-0376), Office of Naval Research(Contract ONR N00014-96-1-0638), and the Department of Energy (Grant DE-FG03-98DP00212). Fig. 4(c) was obtained from a collaborative research with Professor $\mathrm{Y} . \mathrm{Xu}$, and his effort is greatly appreciated. The authors acknowledge discussions and collaboration with a number of colleagues, enabling them to reach this state of understanding: T.W. Wright, U.R. Andrade, H.-r. Pak, C.L. Wittman, Y.B. Xu, J. Shih, Y.J. Chen, H.C. Chen, K.S. Vecchio, S. Nemat-Nasser, J.B. Isaacs, and A.M. Molinari.

\section{References}

[1] F.A. McClintock, A.S. Argon, Mechanical Behavior of Materials, Addison-Wesley, Reading, MA, 1966.

[2] U.F. Kocks, A.S. Argon, M.F. Ashby, 'Thermodynamics and Kinetics of Slip', Oxford: Pergamon Press, 1975.

[3] A.S. Argon, Mechanical properties of single phase crystalline media: deformation at low temperatures, in: R.W. Cahn, P. Haasen (Eds.), Physical Metallurgy, vol. 3, 4th edition, New York, Elsevier Science, 1996, p. 1877.

[4] A.S. Argon, Mechanical properties of single phase crystalline media: deformation at high temperatures, in: R.W. Cahn, P. Haasen (Eds.), Physical metallurgy, vol. 3, 4th edition, New York, Elsevier Science, 1996.

[5] A.S. Argon, Rate processes in plastic deformation of crystalline and non-crystalline solids, in: Mechanics and Materials: Fundamentals and Linkages, Wiley, New York, 1999, p. 175.

[6] D. Deng, A.S. Argon, S. Yip, Phil. Trans. Roy. Soc. Lond. A329 (1989) 613.

[7] V.V. Bulatov, A.S. Argon, Modeling Simul. Mater. Sci. Eng. 2 (1994) 167
[8] V.V. Bulatov, A.S. Argon, Modeling Simul. Mater. Sci. Eng. 2 (1994) 185.

[9] V.V. Bulatov, A.S. Argon, Modeling Simul. Mater. Sci. Eng. 2 (1994) 203.

[10] C.A. Pampillo, H.S. Chen, Mater. Sci. Eng. 13 (1974) 181.

[11] L.W. Meyer, S. Manwaring, in: L.E. Murr, K.P. Staudhammer, M.A. Meyers (Eds.), Metallurgical Applications of Shock-Wave and High-Strain-Rate Phenomena, Dekker, New York, 1986, p. 657.

[12] J.H. Beatty L.W. Meyer M.A. Meyers S. Nemat-Nasser in: Shock-wave and High-Strain Rate Phenomena in Materials Dekker New York 1992, p. 645.

[13] M.A. Meyers, L.W. Meyer, J. Beatty, U. Andrade, K.S. Vecchio, A.H. Chokshi, in: M.A. Meyers, L.E. Murr, K.P. Staudhammer (Eds.), Shock-Wave and High-Strain-Rate Phenomena in Materials, Dekker, New York, 1992, p. 529.

[14] U.R. Andrade, M.A. Meyers, K.S. Vecchio, A.H. Chokshi, Acta Metall. Mater. 42 (1994) 3183.

[15] M.A. Meyers, U. Andrade, A.H. Chokshi, Metall. Mat. Trans. A 26A (1995) 2881.

[16] M.A. Meyers, G. Subhash, B.K. Kad, L. Prasad, Mech. Mater. 17 (1994) 175.

[17] M.A. Meyers, Y.-J. Chen, F.D.S. Marquis, D.S. Kim, Metall. Mater. Trans. 26A (1995) 2493.

[18] V.F. Nesterenko, M.A. Meyers, J.C. LaSalvia, M.P. Bondar, Y.-J. Chen, Y.L. Lukyanov, Mater. Sci. Eng. A229 (1997) 23.

[19] Y.-J. Chen, M.A. Meyers, V.F. Nesterenko, Mater. Sci. Eng. A268 (1999) 70

[20] R.W. Chen, K.S. Vecchio, J. de Physique IV 4 (1994) C8-459.

[21] Y.B. Xu, L.B. Zhong, Y.J. Chen, L.T. Shen, Q. Liu, Y.L. Bai, and M.A. Meyers, Mater. Sci. Eng. A 299 (2001) 287.

[22] V.F. Nesterenko, A.N. Lazaridi, S.A. Pershin, Fizika Goreniya Vzryva 25 (1989) 154.

[23] V.F. Nesterenko, M.P. Bondar, I.V Ershov, in: S.C. Schmidt, J.W. Shanner, G.A. Samara and M. Ross (Eds.) High pressure Science and Techonology 1993, AIP Press New York 1994;1173.

[24] H.A. Grebe, H.-R. Pak, M.A. Meyers, Met. Trans. 16a (1985) 711.

[25] M.A. Meyers, H.-R. Pak, Acta Met. 34 (1986) 2493.

[26] S. Nemat-Nasser, J.B. Isaacs, M. Liu, Acta Mat. 46 (1998) 1307.

[27] G.R. Johnson, W.H. Cook, Proc. 7th Int. Symp. Ballistics, Am. Def. Prep. Ass. (ADPA), Netherlands, 1983.

[28] R.E. Reed-Hill, R. Abhaschian, 'Physical Metallurgy Principles', Third edition, PWS Kent: Boston, MA, p. 261.

[29] K. Okazaki, H. Conrad, Met. Trans. A 3A (1972) 2411.

[30] R. Vandermeer, W.D. Snyder Jr., Met. Trans. A 10A (1979) 1031.

[31] V.P. Kraschenko, V.E. Statsenko, Strength of Materials 13 (1981) 213.

[32] S. Weinig, E.S. Machlin, Trans. AIME 209 (1957) 843.

[33] B. Derby, Acta Metall. 39 (1991) 955.

[34] S. White, Nature 244 (1973) 276.

[35] M. Guillope, J.P. Poirier, J. Geoph. Res. 84 (1979) 5557.

[36] S.M. Schmid, M.S. Paterson, J.N. Boland, Tectonophysics 65 (1980) 245.

[37] P.D. Tungatt, F.J. Humphrey, Acta Metall. 32 (1984) 1625.

[38] J. Gil Sevillano, P. van Houtte, E. Aernoudt, Progress in Matls. Sci. 25 (1981) 69.

[39] D.A. Hughes, N. Hansen, Acta Met. 45 (1997) 3871.

[40] D.A. Hughes, A. Kumar, in: E. Chen, et al. (Eds.), Tantalum, TMS-AIME, Warrendale, PA, 1996, p. 257.

[41] J.C. Hynes, K.S. Vecchio, S. Ahzi, Met. Mat. Trans. 29A (1998) 191.

[42] M.A. Meyers, J.C. LaSalvia, V.F. Nesterenko, Y.J. Chen, B.K. Kad, in: T.R. McNelley (Ed.), Recrystallization and Related Phenomena, Rex'96, Monterey, 1997, p. 279.

[43] J.A. Sabato, R.W. Cahn, J. Nuclear Matls. 3 (1961) 115.

[44] Y. Meunier, R. Roux, J. Moureand, in: Shock-Wave and HighStrain Rate Phenomena in Materials, Dekker, New York, 1992 p. 637. 
[45] C.L. Wittman, M.A. Meyers, H.-R. Pak, Met. Trans. A 21A (1990) 707.

[46] A.P. Sutton, R.W. Balluffi, Interfaces in Crystalline Materials, Clarendon Press, Oxford, 1995, p. 474.

[47] P.G. Shewmon, Diffusion in Solids, Second Edition, TMSAIME, Warrendale PA, 1989, p. 31,200.

[48] L.E. Murr, Interfacial Phenomena in Metals and Alloys, Addison-Wesley, Reading, MA, 1975, p. 131.

[49] J.W. Rudnicki, J.R. Rice, J. Mech. Phys. Solids 23 (1975) 371.

[50] I. Vardoulakis, Int. J. Num. Analyt. Meth. Geomech. 4 (1980) 103.

[51] I. Vardoulakis, Int. J. Solids Struct. 17 (1981) 1085.

[52] I.G. Vardoulakis, B. Graf, IUTAM Conf. on Deformation and Failure of Granular Metals, Delft, 1982, p. 485.

[53] G. Scarpelli, D.M. Wood, IUTAM Conf. on Deformation and Failure of Granular Metals, Delft, 1982, p. 473.

[54] I. Vardoulakis, Acta Mechanica 49 (1983) 57.

[55] J. Desrues, J. Lanier, P. Stutz, Engng Fract. Mech. 21 (1985) 909.

[56] H.-B. Mijhlhaus, I. Vardoulakis, Geotechnique 37 (1987) 271.

[57] D. Kolymbas, G. Rombach, Ingenieur-Archiv. 59 (1989) 177.

[58] J.P. Bardet, J. Engng Mech. 117 (1991) 1466.

[59] D.G. Schaeffer, Proc. R. Soc. Lond. A 436 (1992) 217.

[60] A.S. Saada, G.F. Bianchini, L. Liang, Geotechnique 44 (1994) 35.

[61] V.F. Nesterenko, M.A. Meyers, H-C. Chen, Acta Met. 44 (1996) 2017.
[62] H.C. Chen, M.A. Meyers, V.F. Nesterenko, In: S.C. Schmidt, W.C. Tao (Eds.), Shock Compression of Condensed Matter 1995, AIP Press, 1996, p. 607.

[63] C.J. Shih, V.F. Nesterenko, M.A. Meyers, J Appl. Phys. 83 (1998) 4660.

[64] C.J. Shih, M.A. Meyers, V.F. Nesterenko, Acta Mat. 11 (1998) 4037.

[65] A.V. Potapov, C.S. Campbell, Powder Techn. 94 (1997) 109.

[66] V.N. Nesterenko, M.A. Meyers, T.W. Wright, in: L.E. Murr, K.P. Staudhammer, M.A. Meyers (Eds.), Shock-Wave and High-Strain Rate Phenomena, Elsevier, Amsterdam, 1995, p. 397.

[67] S.A. Pershin, V.F. Nesterenko, Combustion, Explosion and Shock Waves, 1989, 752.

[68] D.E. Grady, M.E. Kipp, J. Mech. Phys. Solid 35 (1987) 95.

[69] T.W. Wright, H. Ockendon, Int. Journal of Plasticity 12 (1996) 927.

[70] D.E. Grady, J. of Geophys. Res. 85 (1980) 913.

[71] A. Molinari, J. Mech. Phys. Sol. 45 (1997) 1551.

[72] R.J. Clifton, Material Response to Ultra High Loading Rates, Rep. NMAB-356, NMAB, NAS, Washington, DC, 1979, Ch. 8.

[73] V.F. Nesterenko, M.A. Meyers, T.W. Wright, Acta Mater. 46 (1998) 327.

[74] D. Peirce, R.J. Asaro, A. Needleman, Acta Met. 31 (1984) 1951.

[75] L. Anand, S.R. Kalidindi, Mech. of Mat. 17 (1994) 223.

[76] R.W. Armstrong, F.J. Zerilli, Mech. of Mat. 17 (1994) 319. 